\title{
Veronesean Representations of Moufang Planes
}

\author{
O. Krauss, J. Schillewaert, \& H. Van Maldeghem
}

\begin{abstract}
In 1901 Severi [18] proved that the complex quadric Veronese variety is determined by three algebraic/differential geometric properties. In 1984 Mazzocca and Melone [10] obtained a combinatorial analogue of this result for finite quadric Veronese varieties. We make further abstraction of these properties to characterize Veronesean representations of all the Moufang projective planes defined over a quadratic alternative division algebra over an arbitrary field. In the process, new Veroneseans over a nonperfect field of characteristic 2 (related to purely inseparable field extensions) are found, and their corresponding projective representations of the associated groups studied. We show that these representations are indecomposable, but reducible, and determine their (irreducible) quotient and kernel.
\end{abstract}

\section{Introduction}

The Veronesean representation of the Moufang projective plane $\mathcal{P}(\mathbb{O})$ related to any Cayley-Dickson division algebra $\mathbb{O}$ is the geometry of the real form $E_{6,2}^{28}$ of a group of exceptional type $E_{6}$ [20]. One of its nice features is that it provides a homogeneous description of these projective planes, which otherwise must be defined via the projective closures of the corresponding affine planes (and this places a certain line in a special role). The subplanes of $\mathcal{P}(\mathbb{O})$ related to its subalgebras have induced representations and similar properties. Three of these properties have been used by Mazzocca and Melone [10] to characterize the finite quadric Veroneseans. More generally, the objects satisfying these axioms are called Veronesean caps. Despite many special cases of Veronesean caps have been classified (among which all finite ones), until now, there was no full classification. In the present paper, we present such a classification, and we find besides the "classical" Veronesean caps, also a class related to inseparable field extensions in characteristic 2 (which can also be viewed as quadratic algebras). The classical Veronesean caps include, besides the split form ${ }^{1} A_{2,2}^{(1)}$ of groups of type $A_{2}$, the natural geometries of the mentioned real form of groups of type $E_{6}$, and also the geometries of the real form ${ }^{2}\left(A_{2} \times A_{2}\right)_{2}^{(1)}$ of groups of type $A_{2} \times A_{2}$ and geometries of the real form $A_{5,2}^{(2)}$ of groups of type $A_{5}$.

Received October 7, 2014. Revision received May 4, 2015.

Research of the second author was supported by Marie Curie IEF grant GELATI (EC grant nr. 328178). 
The Veronesean representations of the projective planes related to inseparable field extensions in characteristic 2 possibly have infinite dimension, which slightly complicates the classification. We study the corresponding projective representations of the group $G:=\mathrm{PSL}_{3}(\mathbb{A})$, with $\mathbb{A}$ a nonperfect field of characteristic 2 , in some more detail in Section 7 . We show that they possess a unique invariant subspace $U$, and we give a detailed description of the irreducible representations induced in $U$ and in the quotient with $U$. Comparing this situation with [4], we see that, although $G$ is not a group of mixed type, it behaves much like it, certainly concerning representations.

The results of the present paper, together with the main results of $[14 ; 15]$, provide a common characterization of the varieties related to the second row of the Freudenthal-Tits magic square, both the split and nonsplit versions. This corresponds to a characterization of the class of all "projective planes" defined over quadratic alternative composition algebras (among which for instance the so-called Hjelmslev-Moufang planes-equivalent with geometries and groups of type $\mathrm{E}_{6}$-introduced by Springer and Veldkamp [11]). In the nonsplit case, additionally inseparable cases occur, whereas in the split case, additionally only some other sporadic varieties occur such as those related to the half-spin $D_{5}$-geometry, to the line Grassmannian of projective 4-space and the Segre varieties of the direct product of a projective line with projective spaces of dimensions 2 or 3 .

The first three cells of the second row of the Freudenthal-Tits magic square admit a generalization to higher dimensions (Veronesean representations of projective spaces over quadratic alternative division algebras). These can also be characterized using a similar approach, but we will do this elsewhere. A big part of this is also contained in the first author's thesis [9]. The split case has recently been treated in [16].

\section{Some Notation}

In this paper, the $n$-dimensional projective space over the skew field $\mathbb{K}$ is denoted by $\mathbb{P}^{n}(\mathbb{K})$ (it arises from a right $(n+1)$-dimensional vector space). The projective space arising from a right vector space $V$ is denoted by $\mathbb{P}(V)$. We will use a slightly different notation for the projective plane (space) defined over a quadratic alternative division ring $\mathbb{A}$, which we will write as $\mathcal{P}_{2}(\mathbb{A})\left(\mathcal{P}_{n}(\mathbb{A})\right)$. This should clearly distinguish the geometries we represent (the $\mathcal{P}(\mathbb{A}) \mathrm{s}$ ) and those in which we represent these (the $\mathbb{P}(V) s$ ).

Concerning projective planes, we will use the following terminology. A collineation is a bijection between two projective planes preserving the line sets in both ways (it is allowed that the two planes are identical, and then we have a permutation of the point set inducing a permutation of the line set). A translation line in a projective plane is a line $L$ with the property that the collineations fixing $L$ pointwise and not fixing any point off $L$ act transitively on the set of points off $L$. A projective plane containing a translation line is called a translation plane. A Moufang plane, or a plane satisfying the Moufang condition, is a projective plane in which every line is a translation line. The projective planes 
$\mathcal{P}_{2}(\mathbb{A})$ with $\mathbb{A}$ a quadratic alternative division ring are all Moufang planes. Conversely, every Moufang plane is isomorphic to such a $\mathcal{P}_{2}(\mathbb{A})$ or to a projective plane coordinatized by a skew field.

Concerning projective spaces $\mathbb{P}(V)$, with $V$ a right vector space over some skew field $\mathbb{K}$, we will denote the subspace spanned by a (point) set $S$ with $\langle S\rangle$. We usually view subspaces as sets of points. Also, we will need to consider projections. These are maps with a projection center $U$ and an image $W$, where $U$ and $W$ are complementary subspaces, that is, $U \cap W=\emptyset$ and $\langle U, W\rangle$ is the whole projective space. The projection from $U$ to $W$ maps a point $p \notin U$ to the point $\langle U, p\rangle \cap W$. The projection from $U$ onto $W$ is not defined on the points of $U$, and if we consider a subspace $S$ intersecting $U$, then by the projection of $S$ we mean the subspace $\langle U, S\rangle \cap W$ (so for subspaces, we do not project pointwise since otherwise we always have to avoid the points of $U \cap S$, which only makes the notation cumbersome).

We end this introduction by mentioning that large parts of Section 6 are taken from the $\mathrm{PhD}$ thesis of the first author, who obtained a partial characterization of $\mathcal{V}_{2}(\mathbb{K}, \mathbb{A})$ (mainly for the case of char $\mathbb{K} \neq 2$ ).

\section{Definitions and Statement of the Main Result}

An ovoid $O$ in a possibly infinite-dimensional projective space $\Sigma$ is a set of points of $\Sigma$ such that no line of $\Sigma$ intersects $O$ in more than two points, and for every point $x \in O$, there is a unique hyperplane $\pi$ through $x$ intersecting $O$ in only $x$ and containing all lines through $x$ that meet $O$ in only $x$. The hyperplane $\pi$ is called the tangent hyperplane at $x$ to $O$ and denoted $T_{x}(O)$. The dimension of the ovoid is equal to $-1+\operatorname{dim} \Sigma$ (and this can be infinite).

Let $V$ be a possibly infinite-dimensional right vector space over some skew field $\mathbb{K}$, and let $\mathbb{P}(V)$ be the corresponding projective space. Let $X$ be a spanning point set of $\mathbb{P}(V)$, and let $\Xi$ be a collection of subspaces of $\mathbb{P}(V)$, which we shall refer to as the elliptic spaces of $X$, such that, for any $\xi \in \Xi$, the intersection $\xi \cap X$ is an ovoid $X(\xi)$ in $\xi$ of dimension at least 1 (and then, for $x \in X(\xi)$, we sometimes denote $T_{x}(X(\xi))$ simply by $T_{x}(\xi)$ ). We call $(X, \Xi)$, or briefly $X$, a Veronesean cap if the following properties (V1), (V2), and (V3) hold.

(V1) Any two points $x$ and $y$ of $X$ lie in a unique element of $\Xi$, denoted by $[x, y]$.

(V2) If $\xi_{1}, \xi_{2} \in \Xi$ with $\xi_{1} \neq \xi_{2}$, then $\xi_{1} \cap \xi_{2} \subset X$.

(V3) For every $x \in X$ and every triple of distinct elliptic spaces $\xi_{1}, \xi_{2}, \xi_{3} \in \Xi$ containing $x$, we have $T_{x}\left(\xi_{3}\right) \subseteq\left\langle T_{x}\left(\xi_{1}\right), T_{x}\left(\xi_{2}\right)\right\rangle$.

If $V$ is finite-dimensional, then we say that $(X, \Xi)$ is finite-dimensional. An ovoid that is the intersection of an elliptic space with $X$ will be called an $X$-ovoid. If all $X$-ovoids have the same dimension $d$, then we say that $X$ has subdimension $d$. We will prove in Lemma 4.1 that every Veronesean cap has a subdimension. Also, we will show in Lemma 4.10 that $(X, \Xi)$ is completely determined by $X$ alone, and so we sometimes speak of the Veronesean cap $X$. We shall denote the geometry of $X$ endowed with the $X$-ovoids by $\mathcal{P}(X, \Xi)$. 
The axioms (V1), (V2), and (V3) express elementary properties of the smooth real Veronesean varieties of the projective planes over the alternative real division algebras, that is, the real, complex, quaternion, and octonion algebras.

We now present a class of examples of Veronesean caps.

Let $\mathbb{A}$ be a quadratic alternative division algebra over the field $\mathbb{K}$, where we assume that $\mathbb{K} \subseteq \mathbb{A}$. This means that $\mathbb{A}$ is a (not necessarily associative) division ring, the multiplication is alternative (thus, $(a b) b=a b^{2}$ and $a(a b)=a^{2} b$, for all $a, b \in \mathbb{A}$ ), $\mathbb{K}$ is a subfield of the center of $\mathbb{A}$, and every element $x \in \mathbb{A}$ satisfies a quadratic equation with coefficients in $\mathbb{K}$. Recall that one of the following situations holds [8]:

- $\mathbb{A}=\mathbb{K}$ (and $\operatorname{dim}_{\mathbb{K}} \mathbb{A}=1$ );

- $\mathbb{A}$ is a quadratic Galois extension of $\mathbb{K}$ (and $\operatorname{dim}_{\mathbb{K}} \mathbb{A}=2$ );

- $\mathbb{A}$ is a quaternion division algebra with center $\mathbb{K}$ (and $\operatorname{dim}_{\mathbb{K}} \mathbb{A}=4$ );

- $\mathbb{A}$ is a Cayley-Dickson division algebra with center $\mathbb{K}$ (and $\operatorname{dim}_{\mathbb{K}} \mathbb{A}=8$ );

- $\mathbb{A}$ is a purely inseparable extension of $\mathbb{K}$ in characteristic 2 such that all squares of $\mathbb{A}$ are contained in $\mathbb{K}$ (and either $\operatorname{dim}_{\mathbb{K}} \mathbb{A}$ is infinite or $\operatorname{dim}_{\mathbb{K}} \mathbb{A}=2^{\ell}$ with $\ell \geq 1)$.

In each case there exists a unique involution $\sigma$ on $\mathbb{A}$, which is an antiautomorphism, which is the identity on $\mathbb{K}$ and for which both $a+a^{\sigma}$ and $a a^{\sigma}$ belong to $\mathbb{K}$ (and we call $\sigma$ the standard involution). In fact, every $a \in \mathbb{A}$ then satisfies the quadratic equation $x^{2}-\left(a+a^{\sigma}\right) x+a^{\sigma} a=0$.

Then we can define the following 1-spaces of the vector space $V:=\mathbb{K} \oplus \mathbb{K} \oplus$ $\mathbb{K} \oplus \mathbb{A} \oplus \mathbb{A} \oplus \mathbb{A}:$

- $\mathbb{K}(1,0,0 ; 0,0,0)$;

- $\mathbb{K}\left(x x^{\sigma}, 1,0 ; 0,0, x\right), x \in \mathbb{A}$;

- $\mathbb{K}\left(x x^{\sigma}, y y^{\sigma}, 1 ; y, x^{\sigma}, x y^{\sigma}\right), x, y \in \mathbb{A}$.

The corresponding set $X$ of points of $\mathbb{P}(V)$ is a Veronesean cap of subdimension $\operatorname{dim} \mathbb{A}$ (see Theorem 3 ), which we denote by $\mathcal{V}_{2}(\mathbb{K}, \mathbb{A})$. In fact, if $\mathbb{A}$ is not a purely inseparable extension of $\mathbb{K}$, then $X$ is the standard Veronesean embedding of the projective plane $\mathcal{P}_{2}(\mathbb{A})$ over $\mathbb{A}$ (or, in other words, coordinatized by $\mathbb{A}$ ): either the quadratic Veronesean $(\mathbb{A}=\mathbb{K})$, or the Hermitian Veronesean $(\mathbb{A}$ is a quadratic Galois extension of $\mathbb{K}$ ), or the quaternion Veronesean $(\mathbb{A}$ is a quaternion division algebra with center $\mathbb{K}$ ), or the octonion Veronesean $(\mathbb{A}$ is a CayleyDickson division algebra with center $\mathbb{K}$ ). If $\mathbb{A}$ is an inseparable extension of $\mathbb{K}$, then this is a new type of Veronesean, which is, as far as we know, not yet studied in the literature, and which we shall call an inseparable Veronesean. In all cases, the points of $\mathcal{P}_{2}(\mathbb{A})$ are in bijective correspondence with the elements of $X$, and the lines of $\mathcal{P}_{2}(\mathbb{A})$ correspond with the $X$-ovoids, which are all quadrics of Witt index 1 .

If $\mathbb{A}$ is associative, then we can define the Veronesean $\mathcal{V}_{n}(\mathbb{K}, \mathbb{A})$ of $\mathcal{P}_{n}(\mathbb{A})$ over $\mathbb{K}, n \geq 2$, explaining the index 2 in the notation. We will not be concerned with $\mathcal{V}_{n}(\mathbb{K}, \mathbb{A})$ for $n \neq 2$, but the present paper lays the foundation to characterize $\mathcal{V}_{n}(\mathbb{K}, \mathbb{A})$ with $n$ finite in a similar way, as was done in the finite case in [3]. This will be done elsewhere. 
We can now state our main result.

MAIN Result. Every Veronesean cap is projectively equivalent to $\mathcal{V}_{2}(\mathbb{K}, \mathbb{A})$ for some commutative field $\mathbb{K}$ and some quadratic alternative division algebra $\mathbb{A}$ over $\mathbb{K}$. In particular, the subdimension is either infinite or a power of 2 , and if the characteristic of the underlying field is not 2, then the subdimensions can only take the values $1,2,4,8$.

As an application, we determine the irreducibility of the corresponding projective group representations.

COROLlary. Let $\mathbb{K}, \mathbb{A}$, and $\sigma$ be as before. If either $\sigma$ is nontrivial or the characteristic of $\mathbb{K}$ is unequal to 2 , then the action of $\mathrm{PSL}_{3}(\mathbb{A})$ on $\mathbb{P}(V)$ induced by the action on $\mathcal{V}_{2}(\mathbb{K}, \mathbb{A})$ is irreducible. If the characteristic of $\mathbb{K}$ is equal to 2 and $\sigma$ is trivial, then the action of $\mathrm{PSL}_{3}(\mathbb{A})$ on $\mathbb{P}(V)$ induced by the action on $\mathcal{V}_{2}(\mathbb{K}, \mathbb{A})$ has a unique nontrivial invariant subspace $U$.

In Section 7 we describe the irreducible representations induced in $U$ and in the quotient space $\mathbb{P}(V) / U$.

Some special cases of the main result are already known in the literature. We give a historic overview.

In 1984, Mazzocca and Melone [10] were the first to define Veronesean caps; they defined Veronesean caps $X$ of subdimension 1 over finite fields, where they assumed the $X$-ovoids to be conics (and not just ovals), and they classified them in the case of odd characteristic. Later, in 1991, Hirschfeld and Thas [6] classified in characteristic 2, and in 2004, Thas and Van Maldeghem [19] weakened the assumptions on the $X$-ovoids to ovals and classified. In the meantime, also in 2004 , Cooperstein, Thas, and Van Maldeghem [3] introduced and classified Veronesean caps of subdimension 2 over finite fields. Then, in 2012 and 2013, the last two present authors (JS + HVM) classified Veronesean caps of subdimension 1 and 2 over arbitrary fields in $[12 ; 13]$ by showing that these are automatically the quadric Veronesean varieties $\mathcal{V}_{2}(\mathbb{K}, \mathbb{K})$ and the Hermitian Veronesean varieties $\mathcal{V}_{2}(\mathbb{K}, \mathbb{L})$ (with $\mathbb{L}$ a quadratic Galois extension of $\mathbb{K}$ ), respectively. However, the paper [12] contains an incompleteness, which we rectify in Section 4.4 (basically, the inseparable Veroneseans with subdimension 2 were overlooked; the two last authors thank the first author for noting this error). Finally, in 2014, the first author, in his thesis [9], classified Veronesean caps under some additional conditions; these conditions exclude characteristic 2 , but they allow the quaternion cases for which the center admits only two quadratic classes, and the octonion case assuming, in the terminology of the present paper, the cap has subdimension 8 (see below for precise definitions).

The main difficulty in handling the case of subdimension at least 3 is to deduce that the algebra $\mathbb{A}$ is quadratic over the underlying field $\mathbb{K}$ of the ambient projective space. On the contrary, it is not so hard to construct the algebra (this follows from the fact that the Veronesean cap $X$ endowed with the $X$-ovoids, is a projective plane; see Proposition 4.5 below), and it also follows rather directly 
that $\mathbb{K}$ is contained in the center of $\mathbb{A}$. The crux of the argument to prove the main difficulty is to construct a Veronesean subcap $X^{\prime}$ of subdimension 2 in general position but containing a given Veronesean subcap $X^{\prime \prime}$ of subdimension 1 . Notice that it is relatively standard to construct all Veronesean subcaps of subdimension 1 , but it is much harder to construct a Veronesean subcap of subdimension 2 . The reason is the following. Using $X$, we can construct an André-Bose-Bruck representation of an affine plane $\mathcal{A}$ arising from $\mathcal{P}(X, \Xi)$; this consists of a projective space $\mathbb{P}(W)$ with a hyperplane $H$ in which a spread $\mathcal{S}$ of pairwise complementary subspaces is given (a spread is a partition of the point set in subspaces). The points of $\mathcal{A}$ are the points of $\mathbb{P}(W) \backslash H$, and its lines are the subspaces of $\mathbb{P}(W)$ containing an element of $\mathcal{S}$ as a hyperplane and not contained in $H$. Now, a Veronesean subcap corresponds with a subspace $U$ of $\mathbb{P}(W)$ intersecting $H$ in a hyperplane $H_{U}$ of $U$ and such that the intersection of the elements of $\mathcal{S}$ with $H_{U}$ induces a spread of pairwise complementary subspaces of $H_{U}$. If $U$ is a plane, then it is easy to choose it in such a way that $U_{H}$ is a line not contained in any member of $\mathcal{S}$, and we are done. This is how to construct Veronesean subcaps of subdimension 1 . However, for subdimension $2, H_{U}$ is three-dimensional, and the chances that a random 3-space of $H$ not contained in any member of $\mathcal{S}$ intersects the elements of $\mathcal{S}$ in a spread of lines are zero. Moreover, in order to fully include the inseparable case, we must deal with the possibility of the $X$-ovoids having infinite dimension.

The proof of the main result is organized as follows. In Section 4, we show that every Veronesean cap has a subdimension and that $\mathcal{P}(X, \Xi)$ is a Moufang projective plane. We also review Veronesean caps of subdimensions 1 and 2 (correcting an oversight in [12]). In Section 5, we construct the subcaps that we need, deduce that $\mathbb{K}$ is a field, construct the algebra $\mathbb{A}$, and show that it is quadratic over $\mathbb{K}$, that it is alternative, and that $\mathbb{K}$ is in the center of $\mathbb{A}$. We then show in Section 6 that the cap is projectively equivalent with $\mathcal{V}_{2}(\mathbb{K}, \mathbb{A})$.

We start with proving in the next section that the examples $\mathcal{V}_{2}(\mathbb{K}, \mathbb{A})$ are Veronesean caps with subdimension $\operatorname{dim} \mathbb{A}$.

\section{Veronesean Embeddings are Veronesean Caps}

In the sequel, $\mathbb{K}$ is a field, and $\mathbb{A}$ is a quadratic alternative division algebra over $\mathbb{K}$.

As already mentioned, $\mathcal{V}_{2}(\mathbb{K}, \mathbb{A})$ is an embedding of the projective plane $\mathcal{P}_{2}(\mathbb{A})$ into the projective space $\mathbb{P}(V)$, where $V=\mathbb{K} \oplus \mathbb{K} \oplus \mathbb{K} \oplus \mathbb{A} \oplus \mathbb{A} \oplus \mathbb{A}$, and where the lines of $\mathcal{P}_{2}(\mathbb{A})$ correspond to the elliptic spaces. We can view $\mathcal{P}_{2}(\mathbb{A})$ as the projective completion of the affine plane $\mathcal{A}_{2}(\mathbb{A})$ over $\mathbb{A}$.

The affine plane $\mathcal{A}_{2}(\mathbb{A})$ has $\mathbb{A} \times \mathbb{A}$ as its point set and the following subsets of $\mathbb{A} \times \mathbb{A}$ are called lines:

$$
\begin{aligned}
{[s, t] } & =\{(x, s x+t) \mid x \in \mathbb{A}\} \quad \text { for } s, t \in \mathbb{A}, \\
{[c] } & =c \times \mathbb{K} \quad \text { for } c \in \mathbb{A} .
\end{aligned}
$$


The special cases $[0,0]$ and $[0]$ are called the horizontal axis and vertical axis, respectively. Also, $s$ is called the slope of the line $[s, t]$ for $s, t \in \mathbb{A}$. The vertical axis and all lines parallel with it have by definition the slope $\infty$.

For the projective completion, the point at infinity on the lines of slope $s \in$ $\mathbb{A} \cup\{\infty\}$ will be denoted by $(s)$, and the line at infinity is

$$
[\infty]:=\{(s) \mid s \in \mathbb{A} \cup\{\infty\}\} .
$$

Let $(X, \Xi)$ be the standard Veronesean embedding of $\mathcal{P}_{2}(\mathbb{A})$. Then Theorem 16.3 of [17] carries over to the general case. In particular, the elliptic spaces are obtained by intersecting $X$ with a hyperplane of $\mathbb{P}(V)$. If $\sigma$ is not trivial or $\operatorname{char} \mathbb{K} \neq 2$, then we denote by $[k, \ell, m ; a, b, c], k, \ell, m \in \mathbb{K}, a, b, c \in \mathbb{A}$. the hyperplane

$$
\begin{aligned}
& \left\{\mathbb{K}\left(x_{0}, x_{1}, x_{2} ; y_{0}, y_{1}, y_{2}\right) \in \mathbb{K}^{3} \times \mathbb{A}^{3}: k x_{0}+\ell x_{1}+m x_{2}+a y_{0}^{\sigma}\right. \\
& \left.\quad+y_{0} a^{\sigma}+b y_{1}^{\sigma}+y_{1} b^{\sigma}+c y_{2}^{\sigma}+y_{2} c^{\sigma}=0\right\} .
\end{aligned}
$$

If $\sigma$ is trivial and char $\mathbb{K}=2$, then we denote by $[k, \ell, m], k, \ell, m \in \mathbb{K}$, the hyperplane with point set $\left\{\mathbb{K}\left(x_{0}, x_{1}, x_{2} ; y_{0}, y_{1}, y_{2}\right) \in \mathbb{K}^{3} \times \mathbb{A}^{3}: k x_{0}+\ell x_{1}+m x_{2}=0\right\}$.

THEOREM 3.1. Let us identify the points of the projective completion $\mathcal{P}_{2}(\mathbb{A})$ of $\mathcal{A}_{2}(\mathbb{A})$ with the points of $X$ in the following way:

$$
\begin{aligned}
(x, y) & \mapsto \mathbb{K}\left(x x^{\sigma}, y y^{\sigma}, 1 ; y, x^{\sigma}, x y^{\sigma}\right), \quad x, y \in \mathbb{A}, \\
(s) & \mapsto \mathbb{K}\left(1, s s^{\sigma}, 0 ; 0,0, s^{\sigma}\right), \quad s \in \mathbb{A}, \\
(\infty) & \mapsto \mathbb{K}(0,1,0 ; 0,0,0) .
\end{aligned}
$$

Then the elliptic spaces correspond to the lines of $\mathcal{P}_{2}(\mathbb{A})$ and are determined by intersecting $X$ with a hyperplane given as follows:

$$
\begin{aligned}
{[s, t] } & \mapsto \mathbb{K}\left[s s^{\sigma}, 1, t t^{\sigma} ;-t, t^{\sigma} s,-s^{\sigma}\right], \\
{[c] } & \mapsto \mathbb{K}\left[1,0, s s^{\sigma} ; 0,-s^{\sigma}, 0\right], \\
{[\infty] } & \mapsto \mathbb{K}[0,0,1 ; 0,0,0]
\end{aligned}
$$

if $\sigma$ is nontrivial or if char $\mathbb{K} \neq 2$, and as

$$
\begin{aligned}
{[s, t] } & \mapsto \mathbb{K}\left[s^{2}, 1, t^{2}\right], \\
{[c] } & \mapsto \mathbb{K}\left[1,0, s^{2}\right], \\
{[\infty] } & \mapsto \mathbb{K}[0,0,1]
\end{aligned}
$$

if $\sigma$ is trivial and if $\operatorname{char} \mathbb{K}=2$.

This mapping from $\mathcal{P}_{2}(\mathbb{K})$ to $\mathbb{P}(V)$ will be referred to as the Veronesean map. The given hyperplanes intersecting $X$ in the points of $X$ in an elliptic space will be called the tangent hyperplanes.

Geometric Structure of a Line (see $\$ 16.2$ of [17] for the case $\mathbb{A}=\mathbb{O}$ and $\mathbb{K}=\mathbb{R})$. Consider the line $[\infty]$ of $\mathcal{P}_{2}(\mathbb{A})$. The set of points incident with it is

$$
\left\{\mathbb{K}\left(1, s s^{\sigma}, 0 ; 0,0, s\right) \mid s \in \mathbb{A}\right\} \cup\{\mathbb{K}(1,0,0 ; 0,0,0)\} .
$$


This is the quadric of Witt index 1 defined by the equations $X_{0} X_{1}=$ $Y_{2} Y_{2}^{\sigma}$ and $X_{2}=Y_{0}=Y_{1}=0$, where a generic point of $\mathbb{P}(V)$ has coordinates $\left(X_{0}, X_{1}, X_{2} ; Y_{0}, Y_{1}, Y_{2}\right)$. This description is easily checked to remain true in the inseparable case.

The Collineation Group of $\mathcal{P}_{2}(\mathbb{A})$ ( see $\$ 16.6$ of [17] for the case $\mathbb{A}=\mathbb{O}$ and $\mathbb{K}=\mathbb{R})$. The following permutation leaves the point set of $\mathcal{V}_{2}(\mathbb{K}, \mathbb{A})$ invariant:

$$
\bar{\tau}: V \rightarrow V:\left(X_{0}, X_{1}, X_{2} ; Y_{0}, Y_{1}, Y_{2}\right) \mapsto\left(X_{2}, X_{0}, X_{1} ; Y_{2}, Y_{0}, Y_{1}\right) .
$$

It therefore induces a bijection $\tau$ on $\mathcal{P}_{2}(\mathbb{A})$, which is easily seen to be a collineation, called the triality collineation.

Furthermore, for $a, b \in \mathbb{A}$, we define the map $\tilde{\tau}_{a, b}: \mathbb{K}^{3} \times \mathbb{A}^{3} \rightarrow \mathbb{K}^{3} \times \mathbb{A}^{3}$, which sends $\left(X_{0}, X_{1}, X_{2} ; Y_{0}, Y_{1}, Y_{2}\right)$ to

$$
\begin{gathered}
\left(X_{0}+Y_{1}^{\sigma} a^{\sigma}+a Y_{1}+a a^{\sigma} X_{2}, X_{1}+b Y_{0}^{\sigma}+Y_{0} b^{\sigma}+b b^{\sigma} X_{2}, X_{2}\right. \\
\left.Y_{0}+b X_{2}, Y_{1}+a^{\sigma} X_{2}, Y_{2}+Y_{1}^{\sigma} b^{\sigma}+a Y_{0}^{\sigma}+a b^{\sigma} X_{2}\right)
\end{gathered}
$$

and which induces the translation $\tau_{a, b}$ in $\mathcal{A}_{2}(\mathbb{A})$ given by

$$
\tau_{a, b}:(x, y) \mapsto(x+a, y+b) .
$$

The corresponding collineation of $\mathcal{P}_{2}(\mathbb{A})$ will also be called a translation. The following theorem can be proved exactly as Proposition 17.2 in [17].

THEOREM 3.2. The collineation group $\mathrm{PSL}_{3}(\mathbb{A})$ of $\mathcal{P}_{2}(\mathbb{A})$ generated by the translations $\tau_{a, b}, a, b \in \mathbb{A}$, and their conjugates $\tau_{a, b}^{\tau}$ and $\tau_{a, b}^{\left(\tau^{2}\right)}$ acts transitively on the set of nondegenerate triangles of $\mathcal{P}_{2}(\mathbb{A})$.

Note that $\mathrm{PSL}_{3}(\mathbb{A})$ has a projective representation in $\mathbb{P}(V)$ via the action on $\mathcal{V}_{2}(\mathbb{K}, \mathbb{A})$ as defined by the $\tau_{a, b}$ and their conjugates.

Theorem 3.3. The standard Veronesean embedding $(X, \Xi)$ of $\mathcal{P}_{2}(\mathbb{A})$ is a Veronesean cap with subdimension $k=\operatorname{dim}_{\mathbb{K}} \mathbb{A}$.

Proof. The lines are $\left(\operatorname{dim}_{\mathbb{K}} \mathbb{A}\right)$-dimensional ovoids as just described. Condition (V1) is fulfilled by Theorem 3.1. For (V2), we may use the horizontal axis and the vertical axis due to Theorem 3.2. Their point sets are given by the equations

$$
\left\{\begin{array} { l } 
{ X _ { 0 } X _ { 2 } = Y _ { 1 } Y _ { 1 } ^ { \sigma } , } \\
{ X _ { 1 } = Y _ { 0 } = Y _ { 2 } = 0 , }
\end{array} \text { and } \left\{\begin{array}{l}
X_{1} X_{2}=Y_{0} Y_{0}^{\sigma}, \\
X_{0}=Y_{1}=Y_{2}=0,
\end{array}\right.\right.
$$

respectively. Hence, their space of intersection solely consists of the point $(0,0,1 ; 0,0,0)$ (which corresponds to the point $(0,0)$ of $\mathcal{P}_{2}(\mathbb{A})$ ). We use this point to verify $(\mathrm{V} 3)$. First, note that the tangent space at $(0,0,1 ; 0,0,0)$ to the horizontal axis has equations $X_{0}=X_{1}=Y_{0}=Y_{2}=0$ and the tangent space to the vertical axis at $(0,0)$ has the equations $X_{0}=X_{1}=Y_{1}=Y_{2}=0$; hence, their span $T$ has the equations $X_{0}=X_{1}=Y_{2}=0$. It is easy to see that $T \cap X=$ $(0,0,1 ; 0,0,0)$. So, it suffices to show that, for all $s \in \mathbb{A}$, all points of the elliptic space $\xi_{s} \in \Xi$ corresponding to the line $[s, 0]$ are contained in the subspace 
spanned by $T$ and $\left(1, s s^{\sigma}, 0 ; 0,0, s^{\sigma}\right)$ (the latter corresponds to the point at infinity $(s)$ of the line $[s, 0]$ of $\mathcal{P}(\mathbb{A}))$. But clearly the point $\left(0,0,1 ; s x, x^{\sigma}, 0\right)$ belongs to $T$, and since

$\left(x x^{\sigma}, s s^{\sigma} x x^{\sigma}, 1 ; s x, x^{\sigma}, x x^{\sigma} s^{\sigma}\right)=\left(0,0,1 ; s x, x^{\sigma}, 0\right)+x x^{\sigma}\left(1, s s^{\sigma}, 0 ; 0,0, s^{\sigma}\right)$,

the assertion follows.

\section{Veronesean Caps are Moufang Projective Planes}

In this section, we let $(X, \Xi)$ be an arbitrary Veronesean cap in a projective space $\mathbb{P}(V)$ over the skew field $\mathbb{K}$. We always assume that $X$ spans the projective space $\mathbb{P}(V)$. As already mentioned, the definition of Veronesean cap in [12; $13]$ is slightly different, and one of the goals of this section is to show that the present definition is equivalent to the one in $[12 ; 13]$ and the fact that $\mathcal{P}(X, \Xi)$ is a projective plane. We first prove that every Veronesean cap has a subdimension.

\subsection{The Subdimension of a Veronesean Cap}

If $W_{1} \subseteq W_{2}$ are two subspaces of $\mathbb{P}(V)$, then the codimension of $W_{1}$ in $W_{2}$, denoted by $\operatorname{codim}_{W_{2}} W_{1}$, is the dimension of the factor space $W_{2} / W_{1}$, where $W_{1}$ and $W_{2}$ are viewed as subspaces of $V$. If $\operatorname{dim} W_{2}-\operatorname{dim} W_{1}$ is well defined, then it is equal to $\operatorname{codim}_{W_{2}} W_{1}$.

Lemma 4.1. Every Veronesean cap $(X, \Xi)$ admits a subdimension.

Proof. Suppose for a contradiction that $\xi_{1}$ and $\xi_{2}$ are two elliptic spaces with different dimensions $n_{1}, n_{2}$, respectively ( $n_{1}$ and/or $n_{2}$ may be an infinite cardinal). Since by Axiom (V1) the graph with vertex set $\Xi$, where two vertices are adjacent if the corresponding elliptic spaces share a point, is connected, we may assume that $\xi_{1}$ and $\xi_{2}$ share a point $x \in X$ (by (V2)). Consider an arbitrary elliptic space $\xi_{3}$ through $x$, distinct from $\xi_{1}$ and distinct from $\xi_{2}$, and put $\operatorname{dim} \xi_{3}=n_{3}$. Without loss of generality we may assume $n_{1} \leq n_{2} \leq n_{3}$ and $n_{1}<n_{3}$. Axiom (V3) implies that

$$
\left\langle T_{x}\left(\xi_{1}\right), T_{x}\left(\xi_{2}\right)\right\rangle=\left\langle T_{x}\left(\xi_{2}\right), T_{x}\left(\xi_{3}\right)\right\rangle=\left\langle T_{x}\left(\xi_{3}\right), T_{x}\left(\xi_{1}\right)\right\rangle=: U .
$$

Since $\xi_{i} \cap \xi_{j}=\{x\}, 1 \leq i<j \leq 3$, we have $\operatorname{codim}_{U} T_{x}\left(\xi_{2}\right)=n_{1}-1$ (when considering $\left.U=\left\langle T_{x}\left(\xi_{1}\right), T_{x}\left(\xi_{2}\right)\right\rangle\right)$ and at the same time $\operatorname{codim}_{U} T_{x}\left(\xi_{2}\right)=n_{3}-1$ (when considering $U=\left\langle T_{x}\left(\xi_{3}\right), T_{x}\left(\xi_{2}\right)\right\rangle$ ). Hence, $n_{1}=n_{3}$, a contradiction.

So from now on, we may freely speak about the subdimension of any Veronesean cap. Also, if $x \in X$, then we denote by $T_{x}$ the space generated by any pair of tangent spaces $T_{x}(\xi)$ and $T_{x}\left(\xi^{\prime}\right)$ with $x \in \xi \cap \xi^{\prime}, \xi, \xi^{\prime} \in \Xi$ and $\xi \neq \xi^{\prime}$. Next, we show the equivalence of our definition of Veronesean cap with that in [12;13] and the fact that $(X, \Xi)$ with natural incidence is a projective plane. 


\subsection{The Projective Plane Associated with a Veronesean Cap}

Recall that $\mathcal{P}(X, \Xi)$ is the geometry having the point set $X$ and the line set $\Xi$, endowed with the natural incidence. We will write $\mathcal{P}=\mathcal{P}(X, \Xi)$ for short. Our goal is to show that this is a projective plane that satisfies the Moufang condition. We prove some lemmas to that aim.

We fix an arbitrary elliptic space $\xi \in \Xi$ and a point $x \in X \backslash \xi$. We denote $O=X(\xi)$.

\section{Lemma 4.2. The spaces $T_{x}$ and $\xi$ are disjoint.}

Proof. First, we note that if $\mathbb{K}$ is finite, then this is easy. Indeed, if the subdimension is equal to $d$ (and then $d \in\{1,2\}$ by Result 48 in [5]), then $O$ has $q^{d}+1$ points. Joining these points to $x$ produces $q^{d}+1$ tangent spaces at $x$ of dimension $d$ inside the subspace $T_{x}$, which has dimension $2 d$, and these meet pairwise in just $x$. Hence, they take into account $\left(q^{d}+1\right)\left(q^{d}+q^{d-1}+\cdots+q\right)+1$ points of $T_{x}$. There are no other points. The lemma now follows from (V2).

Now let $\mathbb{K}$ be infinite and suppose for a contradiction that some point $z$ belongs to both $T_{x}$ and $\xi$. Pick two distinct points $p, q \in O$ (and we can assume that $p$, $q, z$ are not collinear $)$; then $T_{x}=\left\langle T_{x}([p, x]), T_{x}([q, x])\right\rangle$. Hence, there is a line $L$ through $z$ intersecting $T_{x}([p, x])$ in a point $u$ and intersecting $T_{x}([q, x])$ in a point $v$. The line $\langle u, x\rangle$ intersects $T_{p}([p, x])$ in a point $a$, and the line $\langle v, x\rangle$ intersects $T_{q}([q, x])$ in a point $b$. Since $|\mathbb{K}|>2$, we find two points $a^{\prime} \in\langle u, x\rangle$ and $b^{\prime} \in\langle v, x\rangle$ such that $z \in\left\langle a^{\prime}, b^{\prime}\right\rangle$ and $a^{\prime} \neq a, b^{\prime} \neq b$. Since $a^{\prime} \notin T_{p}([p, x])$, there are a point $a^{\prime \prime} \in X \backslash\{p\}$ on $\left\langle p, a^{\prime}\right\rangle$ and a point $b^{\prime \prime} \in X \backslash\{q\}$ on $\left\langle q, b^{\prime}\right\rangle$. The line $\left\langle a^{\prime \prime}, b^{\prime \prime}\right\rangle$ belongs to the 3 -space $\left\langle p, q, z, a^{\prime}\right\rangle$; hence, it intersects the plane $\langle p, q, z\rangle$ in some point $z^{\prime}$, which consequently belongs to $\xi$. But $\left\langle a^{\prime \prime}, b^{\prime \prime}\right\rangle \subseteq\left[a^{\prime \prime}, b^{\prime \prime}\right]$, and so by (V2), $z^{\prime}$ belongs to $X$ and hence to $X\left(\left[a^{\prime \prime}, b^{\prime \prime}\right]\right)$. The latter now contains the three collinear points $a^{\prime \prime}, b^{\prime \prime}, z^{\prime}$, a contradiction.

Lemma 4.3. Let $p \in O$ be arbitrary. Then the subspace $\left\langle O, T_{p}\right\rangle$ does not contain any point of $X \backslash O$.

Proof. Put $W=\left\langle O, T_{p}\right\rangle$. Suppose for a contradiction that $u \in(W \cap X) \backslash O$. First, note that Lemma 4.2 immediately yields that $O$ is not contained in $T_{p}$; hence, $\xi$ and $T_{p}([p, u])$ intersect in just $p$ and generate $W$. It follows that $\xi$ and $[p, u]$ also generate $W$ and hence intersect in a line. This contradicts (V2) and the fact that $X$ does not contain lines.

We now choose a subspace $\Pi$ containing $T_{x}$ and complementary to $\xi$ (this is possible by Lemma 4.2). Let $\rho$ be the projection map from $\xi$ onto $\Pi$.

Lemma 4.4. The projection $\rho$ is injective on $X \backslash O$.

Proof. Let, for a contradiction, $y, z$ be two distinct points of $X \backslash O$ with $y^{\rho}=z^{\rho}$. Then $\xi$ is a hyperplane of the subspace $\langle\xi, y, z\rangle$. Hence, the elliptic space $[y, z]$ 
intersects $\xi$ in a point of the line $\langle y, z\rangle$, which belongs to $X$ by (V2), contradicting (V1) since then $[y, z]$ would contain three collinear points of $X$.

We can now prove our first goal.

Proposition 4.5. The geometry $\mathcal{P}$ is a projective plane. Also, $\Pi=T_{x}$, and so $\xi$ and $T_{x}$ are complementary subspaces of $\mathbb{P}(V)$.

Proof. Let $p$ be any point of $O$. By (V3) we have $T_{p}\left(\xi^{\prime}\right)^{\rho}=T_{p}\left(\xi^{\prime \prime}\right)^{\rho}$ for any $\xi^{\prime}, \xi^{\prime \prime} \in \Xi$ with $p \in \xi^{\prime} \cap \xi^{\prime \prime}$ and $\xi^{\prime} \neq \xi \neq \xi^{\prime \prime}$. We denote by $\Pi_{p}$ this common projection. It follows from Lemma 4.3 that $\Pi_{p}$ is a hyperplane in $\xi^{\prime \rho}=: \Pi_{\xi^{\prime}}$ for any $\xi^{\prime} \in \Xi$ and $p \in \xi^{\prime} \neq \xi$, the complement of which is the affine space $\alpha_{\xi^{\prime}}:=$ $\left(X\left(\xi^{\prime}\right) \backslash\{p\}\right)^{\rho}$. We now consider $\xi^{\prime}=[x, p]$. Note that, by our choice of $\Pi$, the subspace $\Pi_{\xi^{\prime}}$ coincides with $T_{x}\left(\xi^{\prime}\right)$. Choose $q \in O$ with $q \neq p$ and denote $\xi^{\prime \prime}=[x, q]$. We define $\alpha_{\xi^{\prime \prime}}, \Pi_{q}$ and $\Pi_{\xi^{\prime \prime}}=T_{x}\left(\xi^{\prime \prime}\right)=\xi^{\prime \prime \rho}$ similarly as for $\xi^{\prime}$ and $p$. Then, by Lemma 4.4, $\alpha_{\xi^{\prime}} \cap \alpha_{\xi^{\prime \prime}}=\left\{\left(\xi^{\prime} \cap \xi^{\prime \prime}\right)^{\rho}\right\}=\{x\}$, and hence $\Pi_{p} \cap \Pi_{q}=\emptyset$. We denote $\Sigma=\left\langle\Pi_{p}, \Pi_{q}\right\rangle$. It is a hyperplane in the subspace $T_{x}$. Also, $\Pi_{p}$ and $\Pi_{\xi^{\prime \prime}}$ are complementary subspaces in $T_{x}$.

Let $z$ be an arbitrary point of $T_{x} \backslash\left(\Pi_{\xi^{\prime}} \cup \Pi_{\xi^{\prime \prime}} \cup \Sigma\right)$. Then the subspace $\left\langle z, \Pi_{p}\right\rangle$ intersects $\Pi_{\xi^{\prime \prime}}$ in a point $z^{\prime \prime}$. Let $u^{\prime \prime} \in X$ be the inverse image under $\rho$ of $z^{\prime \prime}$. Then the projection of $\left[u^{\prime \prime}, p\right]$ clearly coincides with $\left\langle z^{\prime \prime}, \Pi_{p}\right\rangle$, and so $z$ can be written as $u^{\rho}$ with $u \in X\left(\left[u^{\prime \prime}, p\right]\right)$. We claim that $[x, u]$ intersects $\xi$ nontrivially.

Indeed, suppose for a contradiction that $[x, u] \cap \xi=\emptyset$. Since $T_{x}([x, u]) \subseteq T_{x}$, since $u^{\rho}=z \in T_{x}$, and since $[x, u]=\left\langle T_{x}([x, u]), u\right\rangle$, we have $[x, u]^{\rho} \subseteq T_{x}$. Then $T_{x}([x, u])$ is a hyperplane in $[x, u]^{\rho}$ (since the projection $\rho$ induces an isomorphism from $[x, u]$ into $[x, u]^{\rho}$ as $[x, u]$ and $\xi$ are disjoint). This implies that $[x, u]^{\rho}$ and $\Pi_{\xi^{\prime}}$ intersect in a line $L$ containing $x$. This line is not contained in $T_{x}([x, u])$ since $T_{x}([x, u])$ and $\Pi_{\xi^{\prime}}=T_{x}\left(\xi^{\prime}\right)$ are disjoint. Hence, $L$ contains a second point $y$ of $X([x, u]), y \neq x$. By Lemma 4.4, $y$ belongs to $\Pi_{p}$. But this contradicts Lemma 4.3. Hence, our claim is proved.

It then follows that $z$ is contained in $T_{x}([x, u])$, and so every point of $T_{x}\left(\xi^{\prime}\right) \cup T_{x}\left(\xi^{\prime \prime}\right) \cup \Sigma$ is contained in a tangent subspace at $x$ to some $X$-ovoid containing $x$ and intersecting $O$ in a point. Axiom (V3) implies that there is no room for additional tangent spaces; hence, every elliptic space through $x$ meets $\xi$ nontrivially. Since $\xi$ and $x$ were essentially arbitrary, this shows that every pair of elliptic spaces intersects nontrivially and hence $(X, \Xi)$ is a projective plane. It also means that every point of $X \backslash O$ is projected into $T_{x}$, and so $T_{x}$ coincides with $\Pi$.

We now go on and prove that $\mathcal{P}$ is a Moufang plane.

Proposition 4.6. The projective plane $\mathcal{P}$ satisfies the Moufang condition.

Proof. We continue with the notation of the previous proof. We know that every point of $T_{x} \backslash \Sigma$ is the projection of a point $u$ of $X \backslash O$. Let $u \neq x$. Put $\{r\}=[u, x] \cap \xi$. It easily follows that the projection of $T_{r}([u, x])$ coincides with $[u, r]^{\rho} \cap \Sigma$, and so $\left\{T_{a}^{\rho}: a \in O\right\}$ is a spread $\mathcal{S}$ of $\Sigma$. This now implies that the 
projection of $X \backslash \xi$ onto $\Pi$ is the André-Bruck-Bose representation [1;2] of the affine plane corresponding to $\mathcal{P} \backslash O$. Hence, $O$ is a translation line of $\mathcal{P}$. Since $O$ was arbitrary, the assertion follows.

A consequence of the André-Bruck-Bose representation in the proof of Proposition 4.6 is the following.

COROLlary 4.7. Every point of $\mathbb{P}(V)$ not contained in an elliptic space is contained in the span of two elliptic spaces, one of which can be chosen arbitrarily.

Proof. Let $r$ be an arbitrary point of $\mathbb{P}(V)$ not contained in any elliptic space. With the previous notation, either $r^{\rho}$ is contained in $\Sigma$ or coincides with the projection of some point $z \in X \backslash O$.

- In the former case, $r^{\rho}$ is contained in the projection of $T_{p}, p \in O$, for a unique point $p \in O$; hence, $r$ is contained in the span of $\xi$ and $\xi^{\prime}$, where $\xi^{\prime}$ is an arbitrary elliptic space through $p$ distinct from $\xi$.

- In the latter case, $r$ is contained in the span of $\xi$ and any elliptic space through $z$.

The lemma is proved.

Another consequence is the following.

COROLlary 4.8. Let $\xi_{1}, \xi_{2}, \xi_{3}$ be three elliptic spaces not containing a common point. Then $\left\langle\xi_{1}, \xi_{2}\right\rangle \cap \xi_{3}$ coincides with the line $\left\langle\xi_{1} \cap \xi_{3}, \xi_{2} \cap \xi_{3}\right\rangle$, and $\left\langle\xi_{1}, \xi_{2}, \xi_{3}\right\rangle=$ $\mathbb{P}(V)$.

Proof. This follows immediately from the fact that, identifying $X\left(\xi_{3}\right)$ with $O$, the projections $\xi_{1}^{\rho}$ and $\xi_{2}^{\rho}$ have only one point in common and together span $T_{x}$.

We now review two special cases.

\subsection{Veronesean Caps with Subdimensions 1}

If the subdimension of the Veronesean cap $(X, \Xi)$ is equal to 1 , then the $X$-ovoids are plane ovals. By [13] $X$ is projectively equivalent to a quadric Veronesean variety, that is, a Veronesean cap $\mathcal{V}_{2}(\mathbb{K}, \mathbb{K})$ in $\mathbb{P}^{5}(\mathbb{K})$, where $\mathbb{K}$ is a commutative field. The geometry $\mathcal{P}(X, \Xi)$ is a projective plane isomorphic to $\mathcal{P}_{2}(\mathbb{K})$. The automorphism groups of the cap $(X, \Xi)$ (as a subgroup of the automorphism group of $\mathbb{P}(V)$ ) and of the projective plane $\mathcal{P}_{2}(\mathbb{K})$ are isomorphic in a natural way. We record this and provide a proof.

Proposition 4.9. Every collineation of $\mathcal{P}_{2}(\mathbb{K})$ induces a unique collineation of $\mathbb{P}^{5}(\mathbb{K})$ stabilizing $(X, \Xi)$. Conversely, every collineation of $\mathbb{P}^{5}(\mathbb{K})$ stabilizing $(X, \Xi)$ is induced by a unique collineation of $\mathcal{P}_{2}(\mathbb{K})$. If moreover $|\mathbb{K}|>2$, then every collineation of $\mathbb{P}^{5}(\mathbb{K})$ stabilizing $X$ automatically stabilizes $\Xi$.

Proof. In the case of subdimension 1, the Veronesean map maps the point $\left(x_{0}, x_{1}, x_{2}\right)$ to $\left(x_{0}^{2}, x_{1}^{2}, x_{2}^{2}, x_{1} x_{2}, x_{2} x_{0}, x_{0} x_{1}\right)$. Clearly, these coordinates can be written as the upper triangular part of the symmetric matrix $\left(\begin{array}{lll}x_{0} & x_{1} & x_{2}\end{array}\right)^{T}\left(\begin{array}{lll}x_{0} & x_{1} & x_{2}\end{array}\right)$, 
and we denote the $(i, j)$-entry by $x_{i j}$. Let $M=\left(m_{i j}\right)_{0 \leq i, j \leq 2}$ be a nonsingular matrix over $\mathbb{K}$, and let $\theta$ be an automorphism of $\mathbb{K}$. Then the collineation sending $\left(\begin{array}{lll}x_{0} & x_{1} & x_{2}\end{array}\right)$ to $\left(x_{0}^{\theta} x_{1}^{\theta} x_{2}^{\theta}\right) M$ induces the mapping

$$
\left(\begin{array}{lll}
x_{0} & x_{1} & x_{2}
\end{array}\right)^{T}\left(\begin{array}{lll}
x_{0} & x_{1} & x_{2}
\end{array}\right) \mapsto M^{T}\left(\begin{array}{lll}
x_{0}^{\theta} & x_{1}^{\theta} & x_{2}^{\theta}
\end{array}\right)^{T}\left(x_{0}^{\theta} x_{1}^{\theta} x_{2}^{\theta}\right) M,
$$

which can be written as

$$
x_{i j} \mapsto \sum_{k=0}^{2} \sum_{\ell=0}^{2} m_{k i} x_{k \ell}^{\theta} m_{\ell j} .
$$

Since this expression is symmetric in $i$ and $j$, it can be viewed as a semilinear permutation of the vector space of all symmetric $3 \times 3$ matrices over $\mathbb{K}$, which is in a natural way isomorphic to $\mathbb{K}^{6}$, and by construction it preserves $\mathcal{V}_{2}(\mathbb{K}, \mathbb{K}) \cong$ $(X, \Xi)$. Also, we claim that the corresponding collineation of $\mathbb{P}^{5}(\mathbb{K})$ is unique. Indeed, if not, then there would a nontrivial collineation $\alpha$ of $\mathbb{P}^{5}(\mathbb{K})$ fixing $X$ pointwise. But $X$ clearly contains a skeleton (a set of seven points with no six in the same hyperplane); hence, the associated matrix of $\alpha$ is trivial. Since $X$ contains plane conics, also the corresponding field automorphism is trivial, and hence $\alpha$ is trivial after all.

The converse follows from the fundamental theorem of projective geometry applied to $\mathcal{P}_{2}(\mathbb{K}) \cong(X, \Xi)$. Also, if $|\mathbb{K}|>2$, then the members of $\Xi$ are geometrically characterized as the only planes containing at least four points $p_{1}, p_{2}$, $q_{1}, q_{2}$ of $X$; indeed, if these points would be contained in a plane not belonging to $\Xi$, then the intersection $x=\left\langle p_{1}, p_{2}\right\rangle \cap\left\langle q_{1}, q_{2}\right\rangle$ would belong to two distinct members of $\Xi$, but not to $X$, contradicting (V2).

The last argument of the previous proof can be used to show the following general result.

COROLlARY 4.10. If $|\mathbb{K}|>2$, then every Veronesean cap of subdimension 1 is determined by its point set.

Proof. It suffices to show that every quadruple of points $p_{1}, p_{2}, q_{1}, q_{2} \in X$ in a plane is contained in an elliptic space. This follows as above: if these points would be contained in a plane not belonging to a member of $\Xi$, then the intersection $x=\left\langle p_{1}, p_{2}\right\rangle \cap\left\langle q_{1}, q_{2}\right\rangle$ would belong to two distinct members of $\Xi$, but not to $X$, contradicting (V2).

\subsection{Veronesean Caps with Subdimension 2}

If the subdimension of the Veronesean cap $(X, \Xi)$ is equal to 2 , then the $X$-ovoids are ovoids in projective 3 -spaces. In the classification carried out in [12], in particular in Section 3.2, the authors determine the equation of a generic $X$-ovoid, as an elliptic quadric in $\mathbb{P}_{3}(\mathbb{K}), \mathbb{K}$ commutative, containing two conics $C_{1}, C_{2}$ sharing two distinct points $x_{1}, x_{2}$. The tangent lines at $x_{1}$ and $x_{2}$ to $C_{i}$ intersect in the point $z_{i}, i=1,2$. In [12], we coordinatize $\mathbb{P}_{3}(\mathbb{K})$ in such a way that $x_{1}$ has coordinates $(1,0,0,0), x_{2}$ is $(0,0,1,0), z_{1}$ is $(0,1,0,0)$, and $z_{2}$ is $(0,0,0,1)$. Furthermore, 
we assume that $C_{1}$ contains the point $(1,1,1,0)$, which can be done, but we also assumed in [12] that $C_{2}$ contains the point $(1,0,1,1)$, which is not necessarily true since the choice of the point $(1,1,1,0)$ uniquely determines the first three coordinates of any point (up to a nonzero scalar, though), and hence we should rather consider the coordinates $(1,0, t, 1)$. It follows that $C_{1}$ has the equations

$$
\left\{\begin{array}{l}
X_{1}^{2}=X_{0} X_{2}, \\
X_{3}=0,
\end{array}\right.
$$

whereas $C_{2}$ has the equations

$$
\left\{\begin{array}{l}
t X_{3}^{2}=X_{0} X_{2}, \\
X_{1}=0
\end{array}\right.
$$

Hence, the equation of a generic quadric through $C_{1}$ and $C_{2}$, distinct from the union of the two planes $\pi_{1}$ and $\pi_{2}$, is $X_{1}^{2}+k X_{1} X_{3}+t X_{3}^{2}=X_{0} X_{2}$ with $k \in \mathbb{K}$. The rest of the argument in [12] shows that $k \in \mathbb{K}$ is such that the quadric with equation $X_{1}^{2}+k X_{1} X_{3}+t X_{3}^{2}=X_{0} X_{2}$ is elliptic and isomorphic to every $X$-ovoid. It follows that $X_{1}^{2}+k X_{1} X_{3}+t X_{3}^{2}$ is anisotropic, which means that either this quadratic form defines a unique Galois extension $\mathbb{L}$ of $\mathbb{K}$ giving rise to the Hermitian Veronesean cap $\mathcal{V}_{2}(\mathbb{K}, \mathbb{L})$ of subdimension 2 , or the characteristic of $\mathbb{K}$ is equal to $2, k=0$, and $t$ is a nonsquare in $\mathbb{K}$. The latter gives rise to a purely inseparable quadratic extension $\mathbb{K}^{\prime}=\mathbb{K}(\sqrt{t})$ of $\mathbb{K}$, and we obtain the inseparable Veronesean cap $\mathcal{V}_{2}\left(\mathbb{K}, \mathbb{K}^{\prime}\right)$ of subdimension 2 . Note that our arguments in Section 6 also apply to finish the argument after the previous equations are established, but in [12], the authors refer to [3] (which also works).

\section{The Quadratic Alternative Division Algebra $\mathbb{A}$}

We now embark on the proof of the main result. In this section we show that, with previous notation, the projective plane $\mathcal{P}$ is coordinatized by a quadratic alternative division algebra over $\mathbb{K}$ with $\mathbb{K}$ in the center. Since the cases $d=1,2$ are dealt with in [12; 13], we may assume that $d \geq 3$. Consequently, by [5] (result 48 on page 48 ), we may assume that $\mathbb{K}$ is infinite.

\subsection{General Preliminary Facts}

We take up again the notation of the previous chapter. So $\xi \in \Xi, O=X(\xi)$, and $\rho$ is the projection map from $\xi$ onto a complementary space $\Pi$, which we may identify with $T_{x}$ for any point $x \in X \backslash O$ (and we fix such a point). Recall that $\rho$ is injective on $X \backslash O$. We have chosen two distinct points $p, q \in O$. We also use the notation $\mathcal{S}$ introduced in the proof of Lemma 4.3. Also, recall that we have shown the following (see Proposition 4.6):

FACT 1 . The plane $\mathcal{P}$ is a Moufang plane. 
The inverse image of $\Sigma$ under $\rho$ is a hyperplane of $\mathbb{P}(V)$, which we will denote by $T(\xi)$ or $T(O)$ and refer to as the tangent space to $X$ at $\xi$ or at $O$. Clearly, it intersects $X$ in $O$.

Since $T_{p} \subset T(O), T_{p} \cap X=\{p\}$. Also, since $\Pi_{p}$ and $\Pi_{q}$ are disjoint, we see that the spaces $T_{p}$ and $\xi^{\prime}$ for $\xi^{\prime} \ni q$ and $\xi^{\prime} \neq \xi$ generate $\mathbb{P}(V)$ and so are complementary (this also follows by interchanging the roles of $x$ and $p$ and of $\xi$ and $\xi^{\prime}$ ).

Now let $a \in X \backslash\{x\}$. The space $[a, x]$ meets $T_{x}$ in $T_{x}([a, x])$, and so the projection of $a$ from $T_{x}$ onto $\xi$ coincides with the unique intersection point $O \cap[a, x]$. This implies that the image of the projection of $X \backslash\{x\}$ from $T(x)$ onto $\xi$ coincides with $O$. Denote the projection operator by $\tilde{\rho}$ for further reference. So the image under $\tilde{\rho}$ of any member of $\Xi$ not containing $x$ coincides with $O$. In fact, $\tilde{\rho}$ induces a perspectivity between any pair of lines of $\mathcal{P}$ not incident with the point corresponding to $x$. By varying $x$ we deduce the following:

FACT 2. All members of $\Xi$ are projectively equivalent. For every $\xi \in \Xi$, the ovoid $X(\xi)$ is 3-transitive.

Note that $\rho$ and $\tilde{\rho}$ are in a certain sense "opposite". Indeed, since we have chosen $\Pi=T_{x}$, the kernel of one projection is the image of the other.

In the last paragraph of the proof of Proposition 4.5, we established that every point of $T_{x} \backslash \Sigma$ is contained in the tangent space at $x$ of some $X$-ovoid through $x$. So we have the following fact.

FACT 3. The tangent spaces at $x$ cover the whole tangent space $T_{x}$.

\subsection{Constructing Veronesean Subcaps with Subdimension 1}

Now we construct a subset of $X$ that will turn out to be a quadric Veronesean variety. The construction is completely similar to the one used to classify Hermitian Veronesean caps (see [12]), but we go through the construction again since we need it for the sequel.

Consider a plane $\pi$ in $T_{x}$ through $x$ intersecting $\Sigma$ in a line $K^{\infty}$ (which we call the "line at infinity") not belonging to a spread element (of $\mathcal{S}$ ). Let $L$ be a line in $\pi$ through $x$. Then $L$ intersects $\Sigma$ in a point $u_{L}$, which belongs to some subspace $\Pi_{y_{L}} \in \mathcal{S}$, with $y_{L} \in O$ uniquely determined. The inverse image under $\rho$ of $L \backslash\left\{u_{L}\right\}$ is the intersection of $\left[x, y_{L}\right]$ with $\langle O, L\rangle \subseteq\left\langle O,\left[x, y_{L}\right]\right\rangle$. A dimension argument implies that the inverse image $C_{L}^{\prime}$ under $\rho$ of $L \backslash\left\{u_{L}\right\}$ is the intersection of $X\left[x, y_{L}\right] \backslash\left\{y_{L}\right\}$ with the plane $\left\langle x, u_{L}, y_{L}\right\rangle$. Then $C_{L}=C_{L}^{\prime} \cup\left\{y_{L}\right\}$ is an oval to which the line $u_{L} y_{L}$ is tangent at $y_{L}$. Let $M$ be any line in $\pi$ not containing $x$ and not contained in $\Sigma$. Choose a point $z^{\prime}$ on $M \backslash \Sigma$ and let $z \in X$ be such that $z^{\rho}=z^{\prime}$. Then we can apply the same procedure to $M$ in order to find the inverse image of $M \backslash\{v\}$, where $v=M \cap \Sigma$, and we also obtain an oval $C_{M}$. If we do this for three lines not through $x$ and not having a common intersection point, then we find that the three corresponding ovals span a 5-space $\Lambda$ containing all the ovals $C_{L}$. Let $C^{\infty}$ be the set of points $y_{L}$ for $L$ ranging through the set of lines of $\pi$ through $x$. 
Let $\mathcal{V}$ be the union of all $C_{L}$. Fix an arbitrary oval $C_{L}$ with $x \in L$. We project $\mathcal{V}$ inside $\Lambda$ from the line $x y_{L}$ onto a complementary 3-space $W$. Then the ovals through $x$ are projected onto a set $R_{1}$ of disjoint lines of $W$, and the same happens for the ovals through $y_{L}$; they are projected onto a set $R_{2}$ of disjoint lines. Now one sees that $R_{1}$ is a regulus of a ruled quadric for which $R_{2}$ is the other regulus except for one line $N$. The line $N$ has to be the projection of $C^{\infty} \backslash\left\{y_{\infty}\right\}$, showing that $C^{\infty}$ is contained in a plane inside $\langle O\rangle$, and is hence also an oval (on $O$ ). Consequently, we obtain a system of ovals in $\Lambda$ with the structure of a projective plane. By Theorem 2.2 of [13], $\mathcal{V}$ is a quadric Veronesean over the commutative field $\mathbb{K}$ and is hence a subplane of $\mathcal{P}$. Also note that the injectivity of $\rho$ readily implies that $\langle\mathcal{V}\rangle \cap X=\mathcal{V}$. We record all this for further reference.

FACT 4. The inverse image on $X$ of every affine plane in $T_{x} \backslash \Sigma$ whose line at infinity $K^{\infty}$ is not contained in a member of $\mathcal{S}$ spans a 5 -space $\Lambda$ and is, completed with the oval $C^{\infty}=\Lambda \cap O$, isomorphic to a quadric Veronesean. The oval $C^{\infty}$ coincides precisely with the set of points $z \in O$ with the property $T_{z} \cap$ $K^{\infty} \neq \emptyset$. Also, $\langle\mathcal{V}\rangle \cap X=\mathcal{V}$.

Note also that $\mathcal{V}$ is determined by any two of its conics (since these correspond to lines in an appropriate projection defining a unique plane in that projection). We will call every Veronesean subcap $\mathcal{V}$ of subdimension 1 constructed like this a generic subcap of the first kind.

\subsection{Constructing Veronesean Subcaps with Subdimension 2 Containing $\mathcal{V}$}

We continue with the same notation, except that we will not use $p$ and $q$ on $O$ anymore. Also, we will frequently refer to objects in $\Sigma$ as "at infinity". Since $\mathcal{P}$ is a Moufang plane, it is coordinatized by an alternative division ring $\mathbb{A}$, and since we have a subplane isomorphic to $\mathcal{P}_{2}(\mathbb{K})$, the division ring $\mathbb{A}$ may be assumed to contain $\mathbb{K}$. Next, we want to show that every element of $\mathbb{A}$ is quadratic over $\mathbb{K}$. Therefore, we first describe the coordinatization.

We choose $O$ as the line at infinity and $x$ as the point with coordinates $(0,0)$. The points $(0),(\infty)$ are then chosen on $C^{\infty}$ and $(1,1)$ inside $\mathcal{V}$, essentially arbitrarily. So $\mathbb{K}$ can be identified with the first coordinates of the points $(r, 0)$ inside $\mathcal{V}$. An arbitrary element of $\mathbb{A}$ corresponds to the first coordinate of a point $p=(a, 0)$, and we may assume that $a \notin \mathbb{K}$. The conic of $\mathcal{V}$ corresponding to the line joining $(0,0)$ and $(0)$ is denoted by $C_{0}$; the one corresponding to the line joining $(0,0)$ and $(\infty)$ is denoted by $C_{1}$. The corresponding lines in $\pi$ are denoted by $M_{0}$ and $M_{1}$, respectively. We also set $y=p^{\rho}$.

Our next goal is to include $\mathcal{V}$ together with $p$ in a Hermitian Veronesean (where, for ease of formulation, we view separable Veroneseans with subdimension 2 as Hermitian ones). Such a Veronesean is contained in an 8-space. We first construct a 7-space in it, which we will call $\Omega_{1}$. Then we will extend $\Omega_{1}$ to the 8-space $\Omega_{2}$ we want.

Let $\pi_{1}=\left\langle M_{1}, y\right\rangle$. Then $K_{1}^{\infty}:=\pi_{1} \cap \Sigma$ is not contained in a member of $\mathcal{S}$ since the line $\langle x, y\rangle$ intersects $\Sigma$ in a point of the projection of $T_{(0)}$, and $M_{1}$ intersects 
$\Sigma$ in a point of the projection of $T_{(\infty)}$. Hence, Fact 4 implies that there is some quadric Veronesean $\mathcal{V}_{1}$ in $X$ with a conic $C_{1}^{\infty}$ on $O$ such that $\left(\mathcal{V}_{1} \backslash C_{1}^{\infty}\right)^{\rho}=$ $\pi_{1} \backslash K_{1}^{\infty}$. Let $D$ be the conic on $\mathcal{V}_{1}$ corresponding to the line $\langle x, y\rangle$.

By construction and Fact $4, \Omega=\left\langle\mathcal{V}_{1}\right\rangle \cap\langle\mathcal{V}\rangle$ contains the 3 -space spanned by $C_{1}$ and the point (0). If $\Omega$ were four-dimensional, then it would intersect the plane spanned by any conic $C^{\prime}$ of $\mathcal{V}$ through $x$ distinct from $C_{1}$ and from $C_{0}$, in a line, which is $T_{x}\left(C^{\prime}\right)$ by the last assertion of Fact 4 . Since also $T_{x}\left(C_{1}\right)$ belongs to $\Omega$, axiom (V3) applied to $\mathcal{V}_{1}$ implies $T_{x}\left(C_{0}\right) \subset \Omega$. Consequently, $C_{0} \subset \Omega$. This contradicts $\mathcal{V} \neq \mathcal{V}_{1}$ and the last assertion of Fact 4 . So the space $\Omega_{1}$ generated by $\mathcal{V}$ and $\mathcal{V}_{1}$ is seven-dimensional.

We claim that $\kappa:=\operatorname{dim}\left(\Omega_{1} \cap\langle O\rangle\right)=3$. Indeed, if $\kappa=2$, then $\mathcal{V}$ and $\mathcal{V}_{1}$ would have two conics in common $\left(C_{1}\right.$ and $\left.C^{\infty}\right)$. If $\kappa \geq 4$, then $\Omega_{1} \cap\langle O\rangle$ and the 6-space $\langle\mathcal{V}, D\rangle$ have at least one 3 -space $\Pi$ in common. Then $\Pi$ intersects the 3 -space $\Pi^{\prime}$ spanned by the tangent lines at $x$ to the conics $C_{0}, C_{1}$, and $D$, which is indeed also contained in $\langle\mathcal{V}, D\rangle$, in at least one point $q$, which thus belongs to $\xi$ and to $T_{x}$. This contradicts Lemma 4.2, whence the claim.

Next, we consider an arbitrary line $M_{2}$ in $\pi$ through $x$ but distinct from $M_{0}$ and $M_{1}$. We denote the corresponding conic by $C_{2}$ and the corresponding $X$-ovoid by $\mathrm{O}_{2}$. Let $\mathcal{V}_{2}$ be the quadric Veronesean corresponding to the plane $\left\langle M_{2}, y\right\rangle$ and note $D \subset \mathcal{V}_{2}$. Then, just as before, $\left\langle\mathcal{V}_{2}\right\rangle$ intersects $\left\langle\mathcal{V}_{1}\right\rangle$ and $\langle\mathcal{V}\rangle$ in threedimensional spaces $\Omega_{1}^{\prime}$ and $\Omega^{\prime}$, respectively, containing $x$ and (0). If $\Omega_{1}^{\prime} \cap \Omega^{\prime}$ were a plane (which is contained in $\Omega$ ), then it would intersect the plane $\left\langle C_{1}\right\rangle$ (which is also contained in $\Omega$ ) in a line, which must necessarily be $T_{x}\left(C_{1}\right)$ by the last assertion of Fact 4. Hence, $T_{x}\left(C_{1}\right) \subseteq\left\langle\mathcal{V}_{2}\right\rangle$. Since also $T_{x}\left(C_{2}\right) \subset\left\langle\mathcal{V}_{2}\right\rangle$, it would follow that $T_{x}\left(C_{0}\right) \subset\left\langle\mathcal{V}_{2}\right\rangle$. But since $(0) \in \mathcal{V}_{2}$, this yields $C_{0} \subset \mathcal{V}_{2}$, a contradiction. Hence, $\Omega_{1}^{\prime} \cap \Omega^{\prime}=\langle x,(0)\rangle$. Consequently, $\left\langle\mathcal{V}_{2}\right\rangle=\left\langle\Omega_{1}^{\prime}, \Omega^{\prime}\right\rangle$ (by a dimension argument), and so $\mathcal{V}_{2} \subset \Omega_{1}$. Hence, we have the following:

FACT 5. The inverse image on $X$ with respect to $\rho$ of the affine 3-space $\langle\pi, y\rangle \backslash \Sigma$ is entirely contained in $\Omega_{1}$.

Now we extend $\Omega_{1}$ to the 8 -space $\Omega_{2}$. We use the terminology of a 3-ovoid to mean an ovoid in a 3 -space of some elliptic space such that all points of the ovoid are contained in $X$.

Put $X_{1}=X\left(\Omega_{1}\right)$. Let $Q_{0}=X \cap\left\langle C_{0}, D\right\rangle$ and $Q=X \cap\left\langle C^{\infty}, C_{1}^{\infty}\right\rangle$. Then $Q_{0}$ and $Q$ are 3-ovoids contained in $\Omega_{1}$. Let $O_{1}$ be the $X$-ovoid containing $C_{1}$.

We now first prove a general fact, which we state for the original projection $\rho$, but we will apply it for different projections.

FACT 6. Let $\alpha$ be a plane through $x$ intersecting a member $W$ of $\mathcal{S}$ in a line $L$. Then the inverse image of $\alpha \backslash \Sigma$ under $\rho$, completed with the point $p^{\infty}$ of $O$ corresponding to $W$, is a 3-ovoid $Q$, with $Q=\langle Q\rangle \cap X$.

Proof. Consider a point $p^{\prime \rho} \in \alpha \backslash L$ with $p^{\prime} \in X$. Then $\alpha \backslash L$ is contained in the projection of $X\left(\left[p, p^{\prime}\right]\right)$ from $\langle O\rangle$, which can be considered as the projection of the ovoid $X\left(\left[p, p^{\prime}\right]\right)$ from the point $p$. The assertion now follows easily. 
By Fact 6 every affine plane $\beta$ in $\langle\pi, y\rangle$ with $K_{0}^{\infty}:=\left\langle M_{0}, y\right\rangle \cap \Sigma$ as a line at infinity is the projection of a 3-ovoid $Q_{\beta}$ containing ( 0$)$ and contained in $\Omega_{1}$ by Fact 5. Moreover, the union of all such 3-ovoids is precisely $X \cap \Omega_{1}$, and each of them intersects $C_{1}$ (since each plane $\beta$ intersects $M_{1}$ ).

Consider two affine planes $\beta_{1}, \beta_{2}$ in $\langle\pi, y\rangle$ with $K_{0}^{\infty}$ as a line at infinity. Note that Corollary 4.8 implies that $\left\langle Q_{\beta_{1}}, Q_{\beta_{2}}, C_{1}\right\rangle$ is seven-dimensional and hence coincides with $\Omega_{1}$. This in turn implies that $\Omega_{1} \cap\left\langle O_{1}\right\rangle=\left\langle C_{1}\right\rangle$ is two-dimensional.

We now project $\Omega_{1} \backslash\left\langle O_{1}\right\rangle$ from the elliptic space $\left\langle O_{1}\right\rangle$ onto $T_{(0)}$, and obtain a 4-space $U_{2}$ (since $T_{(0)} \cap\left\langle O_{1}\right\rangle=\emptyset$ ), and we denote the projection map by $\rho_{1}$. Let $\Sigma_{1}:=T\left(O_{1}\right) \cap T_{(0)}$ and put $U_{2}^{\infty}=U_{2} \cap \Sigma_{1}$. Then the projections of all 3-ovoids $Q_{\beta}$ are planes in $U_{2}$ pairwise meeting in $(0)^{\rho_{1}}=(0)$, and they intersect $\Sigma_{1}$ in a set of lines $\mathcal{R}$, which, we claim, forms a regulus.

Indeed, consider the quadric Veroneseans $\mathcal{V}_{r}$ corresponding to the planes $\left\langle r, M_{1}\right\rangle$ of $T_{x}$ with $r \in K_{0}^{\infty}$ arbitrary. Since $\left\langle r, M_{1}\right\rangle$ intersects each plane of $\langle\pi, y\rangle$ through $K_{0}^{\infty}$ in a line not contained in $\Sigma$, we see that $\mathcal{V}_{r}$ contains a conic in every 3-ovoid $Q_{\beta}$ (see above). Since $C_{1}$ belongs to $\mathcal{V}_{r}$, the projection of $\mathcal{V}_{r}$ by $\rho_{1}$ is a plane $\alpha_{r}$, and by the observation just made, $\alpha_{r}$ contains a line of each plane $Q_{\beta}^{\rho_{1}}$. Hence, the line $\alpha_{r} \cap \Sigma_{1}$ intersects every member of $\mathcal{R}$ (and we call such a line an $\mathcal{R}$-transversal). Since $r$ was arbitrary, and since $\mathcal{V}_{r}$ contains $(0)$, all these $\mathcal{R}$-transversals are distinct, and the claim follows.

Now, each point of $U_{2} \backslash \Sigma_{1}$ belongs to the image of $\rho_{1}$. Consequently, by the previous claim, there is a point $s^{\rho_{1}}$ in $U_{2}$ with $s \in X \backslash X_{1}$. Consider the space $\Omega_{2}:=\left\langle s, \Omega_{1}\right\rangle$. We aim to show that $X_{2}=X \cap \Omega_{2}$ is a Veronesean cap with subdimension 2. Note that $\Omega_{2}^{\rho_{1}} \subseteq U_{2}$. We now show the opposite inclusion.

FACT 7. Every point $t \in X$ such that $t^{\rho_{1}} \in U_{2}$ belongs to $X_{2}$. Hence, $\Omega_{2}^{\rho_{1}}=U_{2}$.

Proof. Define the points $s^{\infty}=\left\langle(0), s^{\rho_{1}}\right\rangle \cap \Sigma_{1}$ and $t_{\infty}=\left\langle(0), t^{\rho_{1}}\right\rangle \cap \Sigma_{1}$. We claim that there exists a point $u^{\infty}$ not on any member of $\mathcal{R}$ such that both $\left\langle s^{\infty}, u^{\infty}\right\rangle$ and $\left\langle t^{\infty}, u^{\infty}\right\rangle$ contain two points of the regulus $\mathcal{R}$. (We view $\mathcal{R}$ here as the union of the points of its members; hence, as a hyperbolic quadric.) Indeed, this is trivial if $\left\langle s^{\infty}, t^{\infty}\right\rangle$ intersects $\mathcal{R}$ in two points. If $\left\langle s^{\infty}, t^{\infty}\right\rangle$ is tangent to $\mathcal{R}$, then there is a plane in $U_{2}^{\infty}$ through the line $\left\langle s^{\infty}, t^{\infty}\right\rangle$ intersecting $\mathcal{R}$ in a pair of lines-then any point in that plane off $\left\langle s^{\infty}, t^{\infty}\right\rangle$ will do. Finally, if $\left\langle s^{\infty}, t^{\infty}\right\rangle$ does not intersect $\mathcal{R}$, then every plane in $U_{2}^{\infty}$ through $\left\langle s^{\infty}, t^{\infty}\right\rangle$ intersects $\mathcal{R}$ in a conic $\mathcal{K}$ that, due to our assumption, has neither $s^{\infty}$ nor $t^{\infty}$ as a nucleus. Then the intersection of a secant $S_{s}$ through $s^{\infty}$ with a secant $S_{t}$ through $t^{\infty}$ such that $\left|\left(S_{s} \cup S_{t}\right) \cap \mathcal{K}\right|=4$ does the job (and such secants exist because $|\mathbb{K}|$ is infinite). The claim is proved.

But now the plane $\alpha_{s}=\left\langle(0), s^{\infty}, u^{\infty}\right\rangle$ of $U_{2}$ intersects, by construction, two members of $\mathcal{R}$. Hence, the corresponding quadric Veronesean $\mathcal{V}_{\alpha_{s}}$ contains two conics in $\Omega_{1} \subseteq \Omega_{2}$ and the additional point $s$ of $\Omega_{2}$. So $\mathcal{V}_{\alpha_{s}} \subset \Omega_{2}$; in particular, $u \in \Omega_{2}$. Similarly, the latter implies $t \in \Omega_{2}$. 
FACT 8. Let $u$ be an arbitrary point in $X_{2} \backslash C_{1}$, and $v$ an arbitrary point in $C_{1}$. Then there is a unique 3-ovoid $Q_{u, v} \subseteq X_{2}$ containing both $u$ and $v$. If $u=(0)$, then $Q_{u, v} \subseteq X_{1}$.

Proof. Note that $u^{\rho_{1}} \in U_{2}$. Also, the projection under $\rho_{1}$ of $T_{v}$ contains a unique line $L_{v}$ of $\mathcal{R}$. Then the plane through $L_{v}$ and $u^{\rho_{1}}$ determines the 3-ovoid we were looking for. The other assertion is obvious.

In particular, there is a 3-ovoid $Q_{2}$ in $X_{2}$ containing $C_{2}$ (taking $u \in C_{2} \backslash C_{1}$ and $v=x=C_{1} \cap C_{2}$ ). Let $\tau$ be an arbitrary 3-space in $U_{2}$ through $Q_{2}^{\rho_{1}}$ not containing $(0)^{\rho_{1}}$. Since $\tau \cap \Sigma_{1}$ is a plane $\beta_{1}$, which cannot be contained in $\mathcal{R}$, we find a point of $\left(X_{2} \backslash X_{1}\right)^{\rho_{1}}$ by intersecting $\tau$ with the line $\left\langle(0), c_{1}\right\rangle$ with $c_{1} \notin \beta_{1}$, and not on a member of $\mathcal{R}$. We may assume that this point is $s^{\rho_{1}}$. Since $\beta_{1}$ contains a member, call it $K$, of $\mathcal{R}$, it contains a unique $\mathcal{R}$-transversal $T_{1}$. Note that $K \subseteq T_{x}$. Let $q=C_{2}{ }^{\rho_{1}} \cap K$ and select a point $w$ on $T_{1}$ different from $T_{1} \cap K$. Select a point $z \in K$ different from $q$ and different from $K \cap T_{1}$ such that the plane $\pi^{\prime}:=$ $\left\langle(0), w, z, s^{\rho_{1}}\right\rangle \cap \Sigma_{1}$ does not contain a member of $\mathcal{R}$. (This is possible because there are only two planes in $U_{2}^{\infty}$ through the line $\left\langle w, s^{\rho_{1}},(0)\right\rangle \cap U_{2}^{\infty}$ for which this does not hold.) Now, the plane $\pi_{w, z}:=\left\langle w, z, s^{\rho_{1}}\right\rangle$ is contained in $\tau$ and intersects $Q_{2}^{\rho_{1}}$ in a line $A$ that contains $z$ and intersects $\left(C_{2} \backslash C_{1}\right)^{\rho_{1}}$ in a point $c^{\rho_{1}}$ different from $q$ (with $c \in X_{2}$ ).

The plane $\pi_{w, z}$ determines a quadric Veronesean $\mathcal{V}_{w, z}$, which contains exactly two points of $C_{2}$, namely $c$ and $x$. The line $\left\langle z, c^{\rho_{1}}\right\rangle$, which is contained in $\pi_{w, z}$, is the projection of a conic belonging to $\mathcal{V}_{w, z}$ and lies on $Q_{2}$. Let $e$ be an arbitrary point on $C_{1}$ (corresponding to the member $E$ of $\mathcal{R}$ ). Then $\pi_{w, z} \cap Q_{(0), e}^{\rho_{1}}=\pi_{w, z} \cap$ $\langle(0), E\rangle$ is a point $e^{*}$ (even if $w \in E$ or $E=K$, in which cases $e^{*}=w$ and $e^{*}=z$, respectively; all this follows from the restriction we put on $\pi^{\prime}$ ). Let $E^{*}$ be the set of all such points. If we project $E^{*}$ from $(0)$ onto $\Sigma_{1}$, then the projection is obviously contained in $\mathcal{R}$, and every member (line) of $\mathcal{R}$ contains exactly one point of the projection; on the other hand, it is also contained in $\pi^{\prime}$, which contains $w$ and $z$. Hence, the projection from (0) of $E^{*}$ onto $U_{2}^{\infty}$ is a conic, and since $E^{*}$ is contained in $\pi_{w, z}$, it is itself a conic containing $w$ and $z$. Denote the corresponding set of points of $X_{2}$ by $E_{2}^{*} \subseteq \Omega_{1}$. Note that $E_{2}^{*}$ is an infinite set of points on $\mathcal{V}_{w, z}$ intersecting each conic of $\mathcal{V}_{w, z}$ in at most two points (in fact, it is the intersection of a 4-space in $\left\langle\mathcal{V}_{w, z}\right\rangle$ with $\mathcal{V}_{w, z}$, but we will not need this precise fact).

Now we consider the map $\rho_{2}$ projecting $X \backslash O_{2}$ from $\left\langle O_{2}\right\rangle$ onto $T_{(0)}$ and let $W_{2}:=\Omega_{1}^{\rho_{2}}$. The Veronesean $\mathcal{V}_{w, z}$ projects onto a plane $\pi_{w, z}^{\prime}$ since it has a conic on $Q_{2}$. But $\left(E_{2}^{*}\right)^{\rho_{2}}$ is an infinite set of points in $\pi_{w, z}^{\prime}$ intersecting each line of $\pi_{w, z}^{\prime}$ in at most two points; hence, it certainly contains a triangle and therefore generates $\pi_{w, z}^{\prime}$. But since $E_{2}^{*} \subseteq \Omega_{1}$, this implies that $\pi_{w, z}^{\prime}$ is contained in $W_{2}$. Consequently, $s^{\rho_{2}} \in W_{2}$. This now implies the following:

FACT 9. The set $X_{2}$ is independent of the choice of the conic $C_{1}$ of $\mathcal{V}$ through $x$ and distinct from $C_{0}$. Hence, we may interchange the roles of $C_{1}$ and $C_{2}$. In particular, if $z$ is an arbitrary point in $X_{2} \backslash C_{2}$ and $w$ an arbitrary point in $C_{2}$, then there is a unique 3-ovoid $Q_{w, z}^{\prime} \subseteq X_{2}$ containing both $w$ and $z$. 
Now in the 4-space $U_{2}$, the plane $Q_{2}^{\rho_{1}}$ intersects every plane containing a member of $\mathcal{R} \backslash\{K\}$. Interchanging the roles of $C_{1}$ and $C_{2}$ again, we conclude that every 3-ovoid $Q_{w, z}^{\prime}$ as in Fact 9 intersects $Q_{1}$, with $Q_{1}$ the unique 3-ovoid in $X_{2}$ containing $C_{1}$.

Now let $c_{\infty}$ be an arbitrary point of $U_{2}^{\infty}$ not on a member of $\mathcal{R}$. Let $M$ be a line in $U_{2}$ through $c_{\infty}$ containing a point of $C_{2}^{\rho_{1}}$. Since $M$ contains lots of points off $C_{2}^{\rho_{1}}$, Fact 9 and the argument in the first paragraph after Fact 9 imply that $M$ is contained in the projection $\pi_{M}$ of a 3-ovoid contained in $X_{2}$ (and $\pi_{M}$ is a plane). The intersection $\pi_{M} \cap U_{2}^{\infty}$ is a line $L_{\infty}$ containing $c_{\infty}$ and itself contained in a member of $\mathcal{S}$. Hence, Fact 6 and the arbitrariness of $c_{\infty}$ imply that every pair of points of $X_{2}$ is contained in a unique 3-ovoid contained in $X_{2}$, and $\mathcal{R}$ together with all lines $L_{\infty}$ define a line spread of $U_{2}^{\infty}$ that determines the André-Bruck-Bose representation of a subplane $\mathcal{H}_{a}$ of $\mathcal{P}$. Hence, $X_{2}$, furnished with all 3-ovoids contained in it, is a projective plane. By [12] and Section 4.4, $X_{2}$ is a Hermitian or inseparable Veronesean cap. We call such a subcap a generic subcap of the second kind.

Now $\mathcal{H}_{a}$ contains $\mathcal{V}$ and the point $(a, 0)$ and is coordinatized by a field $\mathbb{L}$ that contains $\mathbb{K}$. Since $\mathcal{H}_{a}$ is a subplane of $\mathcal{P}$, we may view $\mathbb{L}$ as a subalgebra of $\mathbb{A}$. Since the multiplication in $\mathbb{A}$ can be defined geometrically (see Chapter V of [7]), the element $a$ commutes with every element of $\mathbb{K}$. Since $a$ was arbitrary, we see that $\mathbb{K}$ is contained in the center of $\mathbb{A}$. Also, we know by [12] and Section 4.4 that either $\mathbb{L}$ is a quadratic Galois extension of $\mathbb{K}$, or $\mathbb{L}$ has characteristic 2 and $\mathbb{L}$ is a purely inseparable extension of $\mathbb{K}$ with $\mathbb{L}^{2} \subseteq \mathbb{K}$. In both cases, there is a quadratic equation over $\mathbb{K}$ satisfied by $a$. This implies that $\mathbb{A}$ is quadratic over $\mathbb{K}$. Hence, by Theorem 20.3 of [21] and since we may assume that $d \geq 3$, we deduce that either $\mathbb{K}$ is the center of $\mathbb{A}$ and $\mathbb{A}$ is a quaternion or a Cayley-Dickson division algebra, or $\mathbb{K}$ has characteristic 2 and $\mathbb{A}$ is a purely inseparable extension of $\mathbb{K}$ (with $\mathbb{A}^{2} \subseteq \mathbb{K}$ ). We refer to these two different possibilities as the algebraic and the inseparable case, respectively.

\section{Projective Equivalence to $\mathcal{V}_{2}(\mathbb{K}, \mathbb{A})$}

Since $\mathbb{A}$ is a quadratic alternative division algebra over $\mathbb{K}$, there is a standard Veronesean cap $\left(X^{\prime}, \Xi^{\prime}\right)$ in some projective space $\mathbb{P}\left(V^{\prime}\right)$ associated to it, projectively equivalent to $\mathcal{V}_{2}(\mathbb{K}, \mathbb{A})$. Also, $\mathcal{P}\left(X^{\prime}, \Xi^{\prime}\right)$ is isomorphic to $\mathcal{P}$. Hence, there is a natural bijection $\varepsilon: X \rightarrow X^{\prime}$ inducing an isomorphism $\varepsilon: \mathcal{P}(X, \Xi) \rightarrow$ $\mathcal{P}\left(X^{\prime}, \Xi^{\prime}\right)$. We must extend $\varepsilon$ to a collineation $\varepsilon: \mathbb{P}(V) \rightarrow \mathbb{P}\left(V^{\prime}\right)$.

Our first task is to show that both $\varepsilon$ and $\varepsilon^{-1}$ map generic subcaps of the first kind to generic subcaps of the first kind. We denote $\mathcal{P}^{\prime}=\mathcal{P}\left(X^{\prime}, \Xi^{\prime}\right)$.

LEMma 6.1. The bijection $\varepsilon$ and its inverse map generic subcaps of the first kind to generic subcaps of the first kind. 
Proof. With the notation of the previous section, the subcap $\mathcal{V}$ is a generic subcap of the first kind for $(X, \Xi)$. By the definition of $\mathcal{V}_{2}(\mathbb{K}, \mathbb{A})$, however, the image under $\varepsilon$ of $\mathcal{V}$ is the quadric Veronesean subcap $\mathcal{V}_{2}(\mathbb{K}, \mathbb{K})$, which is a generic subcap of the first kind for $\left(X^{\prime}, \Xi^{\prime}\right)$.

Now we define a relation $\sim$ on the set of subplanes of $\mathcal{P}$ as follows. Two subplanes $\Delta$ and $\Delta^{\prime}$ are called elementary adjacent, denoted $\Delta \sim_{e} \Delta^{\prime}$, if they share at least one line and all of its points (this is a point row). The transitive closure of this relation is called the adjacency relation, and two adjacent subplanes $\Delta$ and $\Delta^{\prime}$ are denoted $\Delta \sim \Delta^{\prime}$.

Now let $\Delta$ be a generic subcap of the first kind of $\mathcal{P}$, and suppose that $\Delta^{\prime} \sim_{e} \Delta$. Then $\Delta$ and $\Delta^{\prime}$ share a point row $C$, which corresponds to a plane conic (which we also denote by $C$ ) in $X$. Choose two points $x, y$ in $\Delta^{\prime}$ not in $C$. Then the generic subcap of the first kind determined by $C$ and the unique plane conic through the points $x, y$ and the intersection of the line $x y$ with $C$ in $\Delta^{\prime}$ defines a subplane sharing $C, x, y$; hence, they coincide, and so $\Delta^{\prime}$ is a generic subcap. It follows that every subplane of $\mathcal{P}$ adjacent to $\mathcal{V}$ is a generic subcap of the first kind. Now suppose that $\Delta$ is a generic subcap of the first kind of $\mathcal{P}$. We want to show that $\Delta \sim \mathcal{V}$. By considering a generic subcap $\Delta^{\prime}$ sharing some conic with $\Delta$ and having two points in $\mathcal{V}$, we obtain $\Delta^{\prime} \sim_{e} \Delta$, and hence we may assume that $\Delta$ and $\mathcal{V}$ share at least two points $x, y$. Then, considering a generic subcap $\Delta^{\prime \prime}$ sharing the conic on $\Delta$ through $x$ and $y$ with $\Delta$, and sharing a conic of $\mathcal{V}$ through $x$ not containing $y$, yields $\Delta \sim_{e} \Delta^{\prime \prime} \sim_{e} \mathcal{V}$. So we have shown that the class of subplanes of $\mathcal{P}$ adjacent to $\mathcal{V}$ coincides with the class of generic subcaps of the first kind. Similarly, the class of subplanes of $\mathcal{P}^{\prime}$ adjacent to $\mathcal{V}^{\varepsilon}$ coincides with the class of generic subcaps of the first kind. Since adjacency is defined in a purely geometric way, it is preserved under $\varepsilon$ and $\varepsilon^{-1}$.

The previous lemma says that, if we restrict $\varepsilon$ to an $X$-ovoid, then it maps plane conics to plane conics, and similarly for $\varepsilon^{-1}$. We will now use this to show that the $X$-ovoids are projectively equivalent to the $X^{\prime}$-ovoids. This is the content of the next two lemmas. We phrase the second one more generally using plane ovals. The first one is a slight generalization of the fact that collineations of an affine space uniquely extend to the corresponding projective space. Note that for small finite fields (order up to 4), the second lemma is easily verified since there are unique ovoids in the corresponding 3-space.

Lemma 6.2. Let $x, y\left(x^{\prime}, y^{\prime}\right)$ be two distinct points in a projective space $\Pi\left(\Pi^{\prime}\right)$ with $\operatorname{dim} \Pi \geq 3$ (dim $\left.\Pi^{\prime} \geq 3\right)$, possibly of infinite dimension, and suppose each line of $\Pi\left(\Pi^{\prime}\right)$ contains at least six points. Let $H_{x}$ and $H_{y}\left(H_{x^{\prime}}\right.$ and $\left.H_{y^{\prime}}\right)$ be two hyperplanes in $\Pi\left(\Pi^{\prime}\right)$ containing $x$ and $y\left(x^{\prime}\right.$ and $\left.y^{\prime}\right)$, respectively, but not containing $y$ and $x\left(y^{\prime}\right.$ and $\left.x^{\prime}\right)$, respectively. Put $L=\langle x, y\rangle\left(L^{\prime}=\left\langle x^{\prime}, y^{\prime}\right\rangle\right)$. Set $S=\Pi \backslash\left(H_{x} \cup H_{y} \cup L\right) \cup\{x, y\}\left(S^{\prime}=\Pi^{\prime} \backslash\left(H_{x^{\prime}} \cup H_{y^{\prime}} \cup L^{\prime}\right) \cup\left\{x^{\prime}, y^{\prime}\right\}\right)$. Let $\phi: S \rightarrow$ $S^{\prime}$ be a bijection that preserves collinearity in both directions such that $x^{\phi}=x^{\prime}$ and $y^{\phi}=y^{\prime}$. Then $\phi$ uniquely extends to a collineation $\Pi \rightarrow \Pi^{\prime}$. 
Proof. Let $\mathfrak{L}$ be the set of lines of $\Pi$ containing at least three points of $S$. We call two members of $\mathfrak{L}$ parallel if they intersect in a point off $S$. Let $p \in \Pi \backslash S$. Then we denote by $\mathfrak{L}_{p}$ the subset of $\mathfrak{L}$ of those lines incident with $p$ (in $\Pi$ ). Such a set is a maximal clique of size at least 4 in the graph $G$ with vertex set $\mathfrak{L}$ and adjacency given by being parallel. Conversely, any maximal clique of size at least 4 of $G$ coincides with $\mathfrak{L}_{p}$ for some $p \in \Pi \backslash S$. Indeed, if four lines are mutually parallel, then they must have a common intersection point (off $S$ ) since otherwise one of the lines contains three distinct points of $\Pi \backslash S$, and, consequently, one of the other lines joins two points of either $H_{x}, H_{y}$, or $L$, a contradiction.

We define $\mathfrak{L}^{\prime}$ and $\mathfrak{L}_{p^{\prime}}^{\prime}$ in $\Pi^{\prime}$, with $p^{\prime} \in \Pi^{\prime}$, in a similar way. Now note that $\phi$ induces a bijection $\mathfrak{L} \rightarrow \mathfrak{L}^{\prime}$ since $\phi$ preserves collinearity in both directions. Also, $\phi$ preserves being parallel (in both directions) since two lines $M, N \in \mathfrak{L}$ are parallel precisely when they are disjoint and there exist two other lines $P, Q$ such that each of the pairs $\{P, Q\},\{P, M\},\{P, N\},\{Q, M\},\{Q, N\}$ intersects in a point of $S$ (the existence of $P$ and $Q$ in case $M$ and $N$ are parallel is guaranteed by the fact that each line has at least five points). Consequently, $\phi$ maps maximal cliques of $G$ to maximal cliques of $G^{\prime}$. Hence, we can define the image of a point $p \in \Pi \backslash S$ under $\phi$ as the point $p^{\prime}$ such that $\phi$ maps $\mathfrak{L}_{p}$ to $\mathfrak{L}_{p^{\prime}}^{\prime}$. Note that this is the only possibility in view of the fact that $\phi$ must preserve the set of triples of collinear points $\{p, q, r\}$, where $p, q \in S$ and $r \in \Pi \backslash S$. Hence the uniqueness.

Note also that $\phi$ maps a member of $\mathfrak{L}$ containing $n$ points off $S$ (with $n \in$ $\{1,2,3\}$ ) onto a member of $\mathfrak{L}^{\prime}$ containing $n$ points off $S^{\prime}$ since $n$ is precisely equal to the number of maximal cliques of size at least 4 the corresponding line is contained in.

It remains to show that $\phi$ and its inverse preserve the set of triples of collinear points. So let $a, b, c$ be three collinear points of $\Pi$, and let $M$ be the line containing them. If $M$ contains at least three points of $S$, then the definition of the extension of $\phi$ implies that $a^{\phi}, b^{\phi}$, and $c^{\phi}$ are contained in $M^{\phi}$ (the latter defined via its intersection with $S$ ). Now let $M=L$ and suppose $a \notin\{x, y\}$. Then $\mathfrak{L}_{a}$ has the property that it does not contain any line incident with $x$ or $y$, and every member of it contains at least one point distinct from $a$ not contained in $S$. Since $\mathfrak{L}_{a^{\phi}}^{\prime}$ is a maximal clique in $G^{\prime}$ with similar properties, it follows that $a^{\phi} \in\left\langle x^{\prime}, y^{\prime}\right\rangle$.

Next, suppose $M$ contains $x$ and no other point of $S$, that is, $x \in M \subseteq H_{x}$. Suppose $x \notin\{a, b\}$. Consider a line $N \neq L$ through $x$ with $N \in \mathfrak{L}$ and such that $N$ is contained in the plane $\langle y, M\rangle$. Taking the image under $\phi$ of $N,\langle y, a\rangle,\langle y, b\rangle$, we see that $a^{\phi}$ and $b^{\phi}$ are contained in the plane $\left\langle y^{\prime}, M^{\phi}\right\rangle$, which contains $x^{\prime}$, and hence $x^{\prime}, a^{\phi}, b^{\phi}$ are on the line $\left\langle y^{\prime}, M^{\phi}\right\rangle \cap H_{x^{\prime}}$.

Finally, suppose $M \cap S=\emptyset$. Then we already know that, if $a, b, c \in H_{x}$, then $a^{\phi}, b^{\phi}, c^{\phi} \in H_{x^{\prime}}$ and if $a, b, c \in H_{y}$, then $a^{\phi}, b^{\phi}, c^{\phi} \in H_{y^{\prime}}$. Now we consider a plane $\pi$ through $a, b, c$ not contained in $H_{x} \cup H_{y}$. Let $d$ be a point in $\pi \cap S$, and $N$ a line intersecting $\langle a, d\rangle,\langle b, d\rangle$, and $\langle c, d\rangle$ in three distinct points $a^{*}, b^{*}, c^{*}$, respectively, of $S$. Then clearly $\left\langle a^{\phi}, d^{\phi}\right\rangle,\left\langle b^{\phi}, d^{\phi}\right\rangle$, and $\left\langle c^{\phi}, d^{\phi}\right\rangle$ are coplanar since their union contains the collinear points $a^{* \phi}, b^{* \phi}, c^{* \phi}$. Hence, $a^{\phi}, b^{\phi}, c^{\phi}$ are contained in a line. 
Similarly, $\phi^{-1}$ preserves collinearity, and so $\phi$ is a collineation.

Lemma 6.3. Let $\Pi$ and $\Pi^{\prime}$ be two projective spaces, of possibly infinite dimension $k \geq 3$, which are spanned by ovoids $O$ and $O^{\prime}$, respectively. Suppose each line of $\Pi$ has at least six points. Select two points $x, y \in O$. Suppose $\beta: O \rightarrow O^{\prime}$ is a bijective map that maps intersections of $O$ with planes of $\Pi$ containing $x$ or $y$ to intersections of $O^{\prime}$ with planes of $\Pi^{\prime}$ containing $x^{\beta}$ or $y^{\beta}$, respectively. Then there exists a unique collineation $\phi: \Pi \rightarrow \Pi^{\prime}$ extending $\beta$.

Proof. Define $S=\Pi \backslash\left(T_{x}(O) \cup T_{y}(O) \cup\langle x, y\rangle\right)$, put $x^{\prime}=x^{\beta}, y^{\prime}=y^{\beta}$, and define $S^{\prime}=\Pi^{\prime} \backslash\left(T_{x^{\prime}}\left(O^{\prime}\right) \cup T_{y^{\prime}}\left(O^{\prime}\right) \cup\left\langle x^{\prime}, y^{\prime}\right\rangle\right)$. For a point $a \in S$, we define $\langle x, a\rangle \cap O=$ $\left\{x, a_{x}\right\}$ and $\langle y, a\rangle \cap O=\left\{y, a_{y}\right\}$ with $a_{x} \neq x$ and $a_{y} \neq y$.

We first show the uniqueness. Let $a \in S$. Then, since $k \geq 3$, we can select a point $z \in O$ not in $\langle x, y, a\rangle$. Then $a^{\phi}$ is contained in the intersection of the planes $\left\langle x^{\prime}, z, a_{x}^{\beta}\right\rangle,\left\langle x^{\prime}, y^{\prime}, a_{x}^{\beta}, a_{y}^{\beta}\right\rangle$, and $\left\langle y^{\prime}, z, a_{y}^{\beta}\right\rangle$, which are pairwise different by the choice of $z$, the bijectivity of $\beta$ and the assumption that $\beta$ maps plane intersections to plane intersections. Lemma 6.2 implies the uniqueness of $\phi$.

Now we show the existence. First, let $a$ be contained in $S$. Then, since $x, y$, $a_{x}, a_{y}$ are coplanar, also the images $x^{\beta}, y^{\beta}, a_{x}^{\beta}, a_{y}^{\beta}$ are coplanar, and so we can define $a^{\phi}=\left\langle x^{\beta}, a_{x}^{\beta}\right\rangle \cap\left\langle y^{\beta}, a_{y}^{\beta}\right\rangle$. Clearly, this defines a bijection between $S$ and $S^{\prime}$. We now show that $\phi$ and its inverse preserve collinearity. The argument for the inverse is exactly the same, so we only consider $\phi$.

Let $a, b, c \in S$. There are two possibilities.

Case 1. Suppose that $\operatorname{dim}\langle x, y, a, b, c\rangle=3$. Then the points $x, a_{x}, b_{x}, c_{x}$ are coplanar, and so $a^{\phi}, b^{\phi}, c^{\phi}$ are all contained in the plane $\pi_{x^{\prime}}=\left\langle x^{\prime}, a_{x}^{\beta}, b_{x}^{\beta}, c_{x}^{\beta}\right\rangle$. Likewise, those points are also contained in the plane $\pi_{y^{\prime}}=\left\langle y^{\prime}, a_{y}^{\beta}, b_{y}^{\beta}, c_{y}^{\beta}\right\rangle$. If $\pi_{x^{\prime}}$ were equal to $\pi_{y^{\prime}}$, then the oval $O^{\prime} \cap \pi_{x^{\prime}}$ would be the image under $\beta$ of two distinct ovals on $O$, contradicting the hypothesis on the bijectivity of $\beta$. Hence, $a^{\phi}, b^{\phi}, c^{\phi}$ are contained in $\pi_{x^{\prime}} \cap \pi_{y^{\prime}}$ and hence are collinear.

Case 2. Suppose now $\pi=\langle x, y, a, b, c\rangle$ is a plane. Consider a second though arbitrary plane $\pi_{0} \neq \pi$ through $a, b, c$ and select $z \in\left(S \cap \pi_{0}\right) \backslash \pi$. Note that $z$ is not in the plane $\pi=\left\langle x, a_{x}, b_{x}, c_{x}, y\right\rangle$, and hence $z^{\phi}$ is not in the plane $\left\langle x^{\prime}, a_{x}^{\beta}, b_{x}^{\beta}, c_{x}^{\beta}, y^{\prime}\right\rangle=\left\langle x^{\prime}, a^{\phi}, b^{\phi}, c^{\phi}, y^{\prime}\right\rangle$. Since lines have at least six points, we can find three collinear points $a_{0}, b_{0}, c_{0}$ on $\langle a, z\rangle,\langle b, z\rangle,\langle c, z\rangle$, respectively, such that $\left\langle x, y, a_{0}, b_{0}, c_{0}\right\rangle$ is not a plane. By Case $1, a^{\phi}, a_{0}^{\phi}, z^{\phi}$ are on a line, and so are $b^{\phi}, b_{0}^{\phi}, z^{\phi}$ and $c^{\phi}, c_{0}^{\phi}, z^{\phi}$. Now by the same token, $a_{0}^{\phi}, b_{0}^{\phi}, c_{0}^{\phi}$ are on a line, and so $a^{\phi}, b^{\phi}, c^{\phi}$ are contained in the plane $\left\langle z^{\phi}, a_{0}^{\phi}, b_{0}^{\phi}, c_{0}^{\phi}\right\rangle$, which differs from the plane $\left\langle x^{\prime}, a^{\phi}, b^{\phi}, c^{\phi}, y^{\prime}\right\rangle$, as noticed before. Hence, $a^{\phi}, b^{\phi}, c^{\phi}$ are contained in two distinct planes, and consequently they are collinear.

The assertion now follows from Lemma 6.2.

Due to this lemma, we can extend $\varepsilon$ to all points of all elliptic spaces of $(X, \Xi)$. However, we do not yet know that the resulting map is the restriction of a common 
collineation defined globally on $\mathbb{P}(V)$. Before we can tackle this problem, we prove a lemma.

Lemma 6.4. Let $\mathrm{O}_{1}, \mathrm{O}_{2}, \mathrm{O}_{3}$ be three pairwise different $\mathrm{X}$-ovoids with pairwise different intersection points $x_{i j}=O_{i} \cap O_{j}, 1 \leq i \leq j \leq 3$, and denote $x_{i j}=x_{j i}$. Let $z$ be any point of $X \backslash\left(O_{1} \cup O_{2} \cup O_{3}\right)$. Put $x_{i}=\left[x_{j k}, z\right] \cap O_{i}$ for $\{i, j, k\}=$ $\{1,2,3\}$ and $j \leq k$. Then

$$
\left\langle T_{x_{12}}, x_{3}\right\rangle \cap\left\langle T_{x_{23}}, x_{1}\right\rangle \cap\left\langle T_{x_{13}}, x_{2}\right\rangle=\{z\} .
$$

Proof. Let $\{i, j, k\}=\{1,2,3\}$ and $j \leq k$. Since

$$
z \in\left[x_{j k}, z\right]=\left[x_{j k}, x_{i}\right]=\left\langle T_{x_{j k}}\left(\left[x_{j k}, x_{i}\right]\right), x_{i}\right\rangle \subseteq\left\langle T_{x_{j k}}, x_{i}\right\rangle,
$$

it suffices to show that $\left\langle T_{x_{12}}, x_{3}\right\rangle \cap\left\langle T_{x_{23}}, x_{1}\right\rangle \cap\left\langle T_{x_{13}}, x_{2}\right\rangle$ has dimension at most 0 .

Let $U=\left\langle T_{x_{12}}, x_{3}\right\rangle \cap\left\langle T_{x_{23}}, x_{1}\right\rangle \cap\left\langle T_{x_{13}}, x_{2}\right\rangle$. We claim that $\left\langle T_{x_{12}}, x_{2}, x_{3}\right\rangle \cap$ $\left\langle T_{x_{23}}, x_{1}, x_{2}\right\rangle=\left\langle z, O_{2}\right\rangle$. Indeed, both $\left\langle T_{x_{12}}, x_{2}, x_{3}\right\rangle$ and $\left\langle T_{x_{23}}, x_{1}, x_{2}\right\rangle$ contain $O_{2}$, and hence it suffices to show that, if we denote by $\rho_{2}$ the projection operator from $\left\langle O_{2}\right\rangle$ onto $T_{z}$, then the projection under $\rho_{2}$ of $\left\langle T_{x_{12}}, x_{3}\right\rangle$ intersects the projection of $\left\langle T_{x_{23}}, x_{1}\right\rangle$ in just $z$. But this is true by Lemma 4.4 and since $\left\langle T_{x_{12}}, x_{3}\right\rangle^{\rho_{2}}=$ $\left\langle T_{x_{12}}\left(\left[x_{12}, x_{3}\right]\right), x_{3}\right\rangle^{\rho_{2}}=\left[x_{12}, x_{3}\right]^{\rho_{2}}$ and likewise $\left\langle T_{x_{23}}, x_{1}\right\rangle^{\rho_{2}}=\left[x_{23}, x_{1}\right]^{\rho_{2}}$. Since $\left[x_{12}, x_{3}\right]=\left[x_{12}, z\right]$ and $\left[x_{23}, x_{1}\right]=\left[x_{23}, z\right]$, the claim is proved. Consequently, $U \subseteq\left\langle z, O_{2}\right\rangle$, and similarly, $U \subseteq\left\langle z, O_{1}\right\rangle \cap\left\langle z, O_{2}\right\rangle \cap\left\langle z, O_{3}\right\rangle$. Assume for a contraction that $U$ contains a line $L$. Then $L$ contains a point $\ell$ of $\left\langle O_{1}\right\rangle$, which must also be contained in $\left\langle T_{x_{23}}, x_{1}\right\rangle$, implying $\ell=x_{1}$ in view of $T_{x_{23}} \cap\left\langle O_{1}\right\rangle=\emptyset$ (see Lemma 4.2). Likewise, $x_{2}, x_{3} \in L$. But then the three points $x_{1}, x_{2}, x_{3}$ of $X$ are collinear, a contradiction. Hence, $U=\{z\}$, and the lemma is proved.

Now we extend $\varepsilon$ to the whole of $\mathbb{P}(V)$. The technique will consist in considering three $X$-ovoids, extending $\varepsilon$ restricted to these $X$-ovoids to the elliptic spaces of these $X$-ovoids, then extending that map to a collineation defined on the whole of $\mathbb{P}(V)$, and proving that it coincides with $\varepsilon$ over $X$.

Theorem 6.5. The bijection $\varepsilon: X \rightarrow X^{\prime}$ can be uniquely extended to a collineation $\mathbb{P}(V) \rightarrow \mathbb{P}\left(V^{\prime}\right)$ mapping $X$ onto $X^{\prime}$ and $\Xi$ to $\Xi^{\prime}$.

Proof. It follows from Proposition 4.9 that we may assume that $k>2$. First, we show the uniqueness. Let $\alpha$ and $\gamma$ be two collineations that extend $\varepsilon$. Then by Lemma 6.3, $\alpha$ and $\gamma$ coincide on every elliptic space. Since every two elliptic spaces intersect in a point, it follows that they also coincide on the span $\left\langle\xi_{1}, \xi_{2}\right\rangle$ with $\xi_{1}, \xi_{2} \in \Xi$. Corollary 4.7 concludes the proof.

Next, we show the existence. Let $O_{1}, O_{2}, O_{3}$ be the $X$-ovoids with coordinates $[0,0],[0]$, and $[\infty]$, respectively. Denote $\left\langle O_{i}\right\rangle=: \xi_{i} \in \Xi, i=1,2,3$, and denote $x_{12}:=O_{1} \cap O_{2}, x_{13}:=O_{1} \cap O_{3}$, and $x_{23}:=O_{2} \cap O_{3}$ (slightly different from the notation in Section 5.3, but more systematic and suitable for our purposes here). 
For every $i \in\{1,2,3\}$, there is a collineation $\varepsilon_{i}: \xi_{i} \rightarrow\left\langle O_{i}^{\varepsilon}\right\rangle$ that maps $x \in O_{i}$ to $x^{\varepsilon}$. These collineations are induced by semilinear maps. Denote the corresponding $\mathbb{K}$-automorphisms by $\theta_{i}$. We claim that $\theta_{i}=\theta_{j}$ for $i, j \in\{1,2,3\}$. Indeed, the image of $\mathcal{V}$ under $\varepsilon$ is again a generic subcap of the first kind, and $\varepsilon_{i}$ extends the action of $\varepsilon$ to one of the planes $\pi_{i}$ of $\mathcal{V}, i=1,2,3$. But $\varepsilon_{i}$ restricted to $\pi_{i}$ is a collineation, and it is the unique one extending the action of $\varepsilon$ on the conic $X \cap \pi_{i}, i=1,2,3$. Proposition 4.9 implies that all $\varepsilon_{i}, i=1,2,3$, are the restriction of one global collineation $\langle\mathcal{V}\rangle \rightarrow\left\langle\mathcal{V}^{\varepsilon}\right\rangle$, requiring $\theta_{1}=\theta_{2}=\theta_{3}$. The claim is proved.

By recoordinatizing $V^{\prime}$ according to $\theta_{1}$, or applying $\theta_{1}^{-1}$ to $\mathcal{V}^{\prime}$ (which is globally preserved), we may assume that $\theta_{1}$ is trivial.

Hence, since $\theta_{1}=\theta_{3}=\mathrm{id}$, we can extend $\varepsilon_{1}$ and $\varepsilon_{3}$ to a common map $\varepsilon_{13}$ defined on $\left\langle O_{1}, O_{3}\right\rangle$. By Corollary 4.8 the domains of definition of $\varepsilon_{13}$ and $\varepsilon_{2}$ intersect in the line $L=\left\langle x_{12}, x_{23}\right\rangle$. We claim that they coincide on $L$.

Indeed, let $C_{i}=\mathcal{V} \cap O_{i}, i=1,2,3$. Then $\varepsilon_{13} /\left(C_{1} \cup C_{3}\right)=\varepsilon /\left(C_{1} \cup C_{3}\right)$ and $\varepsilon_{2} / C_{2}=\varepsilon / C_{2}$. Denote the unique extension of $\varepsilon / \mathcal{V}$ to $\langle\mathcal{V}\rangle$ by $\varepsilon^{\prime}$. Since extensions of maps on conics to their planes are unique (as collineations), $\varepsilon_{13} / L=\varepsilon^{\prime} / L$ and $\varepsilon_{2} / L=\varepsilon^{\prime} / L$. Hence, $\varepsilon_{13} / L=\varepsilon_{2} / L$, proving our claim. Consequently, there exists a collineation $\varepsilon_{123}: \mathbb{P}(V)=\left\langle\xi_{1}, \xi_{2}, \xi_{3}\right\rangle \rightarrow \mathbb{P}\left(V^{\prime}\right)$ coinciding with $\varepsilon$ on $O_{1} \cup$ $\mathrm{O}_{2} \cup \mathrm{O}_{3}$.

Next, let $z \in X \backslash\left(O_{1} \cup O_{2} \cup O_{3}\right)$. We show that $z^{\varepsilon}=z^{\varepsilon 123}$. By $x_{i}$ we denote for all $\{i, j, k\}=\{1,2,3\}$ the intersection point $\left[x_{j k}, z\right] \cap L_{i}$. By Lemma 6.4, $z$ is the unique intersection point of $\left\langle T_{x_{12}}, x_{3}\right\rangle,\left\langle T_{x_{23}}, x_{1}\right\rangle$, and $\left\langle T_{x_{13}}, x_{2}\right\rangle$, and $z^{\varepsilon}$ is the unique intersection point of $\left\langle T_{x_{12}^{\varepsilon}}, x_{3}^{\varepsilon}\right\rangle,\left\langle T_{x_{23}^{\varepsilon}}, x_{1}^{\varepsilon}\right\rangle$, and $\left\langle T_{x_{13}^{\varepsilon}}, x_{2}^{\varepsilon}\right\rangle$. Since $\varepsilon_{123}$ is a collineation, we get $z^{\varepsilon}=z^{\varepsilon_{123}}$.

Hence, $\varepsilon_{123}$ maps $X$ to $X^{\prime}$, and Corollary 4.10 implies that $\varepsilon_{123}$ maps $\Xi$ to $\Xi^{\prime}$.

This completes the proof of the main result.

\section{Projective Representations of $\mathrm{PSL}_{3}(\mathbb{A})$}

The traditional Veronesean representation of a projective plane leads to a representation of the automorphism group having three orbits on the projective space $\mathbb{P}(V)$. In the case of the quadric Veronesean in characteristic 2, there are four orbits amongst which an invariant subspace. Hence, in the latter case, the corresponding projective representation of the collineation group is reducible. In this section we determine all invariant subspaces of the projective representations of the collineation groups of $\mathcal{P}_{2}(\mathbb{A})$ generated by the elations and acting on the corresponding Veronesean cap (where $\mathbb{A}$ is as before). We also identify all irreducible projective representations arising from both the invariant subspaces and their quotients. As a corollary, we will deduce that the original representations are indecomposable, though possibly reducible. Our results are very similar to those of [4]. This is explained by the fact that the Veronesean caps discussed here appear as "residues" in the dual polar spaces discussed in [4]. 
We start with the irreducible case.

Proposition 7.1. Let $\mathbb{A}$ be a quadratic alternative division ring over the field $\mathbb{K}$ with standard involution $\sigma$. If either $\sigma$ is nontrivial or the characteristic of $\mathbb{K}$ is unequal to 2 , then the action of $\mathrm{PSL}_{3}(\mathbb{A})$ on $\mathbb{P}(V)$ induced by the action on $\mathcal{V}_{2}(\mathbb{K}, \mathbb{A})$ is irreducible.

Proof. First, let $b \in \mathbb{A}^{\times}$. Then we define $\tilde{\gamma}_{b}=\tilde{\tau}_{b^{-1}, 0} \cdot \tilde{\tau}_{0, b}^{\tau} \cdot \tilde{\tau}_{b^{-1}, 0}$, and a straightforward calculation shows that the product $\tilde{\delta}=\tilde{\gamma}_{b} \tilde{\gamma}_{-1}$ acts as follows:

$$
\begin{aligned}
\tilde{\delta}_{b} & :\left(X_{0}, X_{1}, X_{2} ; Y_{0}, Y_{1}, Y_{2}\right) \\
& \mapsto\left(\left(b b^{\sigma}\right)^{-1} X_{0}, X_{1},\left(b b^{\sigma}\right) X_{2} ; Y_{0} b^{\sigma}, b Y_{1} b^{-\sigma}, b^{-1} Y_{2}\right) .
\end{aligned}
$$

We denote the corresponding projective maps without the tilde. Note that $\tilde{\gamma}_{1}^{\tilde{\tau}} \cdot \tilde{\gamma}_{1}$. $\tilde{\delta}_{-1}$ produces $\tilde{\tau}$; hence, the triality collineation belongs to $\mathrm{PSL}_{3}(\mathbb{A})$.

The proposition will be proved if we show that the orbit of every point of $\mathbb{P}(V)$ under the action of $\mathrm{PSL}_{3}(\mathbb{A})$ generates $\mathbb{P}(V)$. Consider an arbitrary point $p=\mathbb{K}\left(x_{0}, x_{1}, x_{2} ; y_{0}, y_{1}, y_{2}\right)$ of $\mathbb{P}(V)$. Using an appropriate $\tau_{a, b}$ for suitable $a, b \in \mathbb{A}$, we see that we may assume that $\left(x_{0}, x_{1}, x_{2}\right) \neq(0,0,0)$. Up to triality, we may assume that $x_{2} \neq 0$. Then using $\tau_{a, b}$ with $a=-x_{2}^{-\sigma} y_{1}^{\sigma}$ and $b=-y_{0} x_{2}^{-1}$, we obtain a point $p^{\prime}=\mathbb{K}\left(x_{0}^{\prime}, x_{1}^{\prime}, x_{2}^{\prime} ; 0,0, y_{2}^{\prime}\right)$ in the orbit of $p$. Suppose first that $y_{2}^{\prime \prime} \neq 0$. Our assumptions imply that there exist $b, b^{\prime} \in \mathbb{A}$ with $b b^{\sigma}=b^{\prime} b^{\prime \sigma}$ and $b \neq b^{\prime}$. Then the line $p^{\prime \delta_{b}} p^{\prime \delta_{b^{\prime}}}$ contains a point with coordinates $\left(0,0,0 ; 0,0, y_{2}^{\prime \prime}\right)$, which is contained in the subspace generated by the orbit. Using $\delta_{b}$ for $b \in \mathbb{A}^{\times}$ and triality, we deduce that all points $\mathbb{K}\left(0,0,0 ; a_{0}, a_{1}, a_{2}\right), a_{0}, a_{1}, a_{2} \in \mathbb{A}$, belong to the subspace generated by the orbit of $p$. Applying $\tau_{1,0}$ to $\mathbb{K}\left(0,0,0 ; 0, a_{1}, 0\right)$ with $a_{1}+a_{1}^{\sigma} \neq 0$, we see that $\mathbb{K}(1,0,0 ; 0,0,0)$ belongs to that subspace, and then triality completes the proof in this case.

Now suppose that $y_{2}^{\prime \prime}=0$. Using $\delta_{b}$ and its conjugates under the triality collineation for suitable $b$, it is easy to see that all points $\mathbb{K}\left(r_{0}, r_{1}, r_{2} ; 0,0,0\right)$ belong to the space $U$ generated by the orbit of $p$. Applying $\tau_{1,1}$ to $\mathbb{K}(0,0,1 ; 0,0,0)$, we see that $\mathbb{K}(1,1,1 ; 1,1,1)$ belongs to $U$. Since that point belongs to $\mathcal{V}_{2}(\mathbb{K}, \mathbb{A})$, and the latter generates $\mathbb{P}(V)$, we are done.

If the characteristic of $\mathbb{K}$ is equal to 2 and $\sigma=1$, then Proposition 7.1 is false. We introduce two other projective representations of $\mathrm{PSL}_{3}(\mathbb{A})$ in this case. First, we call the representation of $\operatorname{PSL}_{3}(\mathbb{A})$ on $\mathbb{P}(\mathbb{A} \times \mathbb{A} \times \mathbb{A})$, where $\mathbb{A}$ is viewed in the natural way as a vector space over $\mathbb{K}$, the $(\mathbb{K}, \mathbb{A})$-representation of $\mathrm{PSL}_{3}(\mathbb{A})$. Second, we call the representation of $\mathrm{PSL}_{3}(\mathbb{A})$ in $\mathbb{P}(\mathbb{K} \times \mathbb{K} \times \mathbb{K})$ naturally obtained from the inclusion $\mathbb{A}^{2} \subseteq \mathbb{K}$ (where $\mathbb{A}^{2}$ denotes the field of all squares of $\mathbb{A}$ ) the $\mathbb{A}^{2}$-representation of $\mathrm{PSL}_{3}(\mathbb{A})$.

We then have the following proposition.

Proposition 7.2. Let $\mathbb{A}$ either be a purely inseparable quadratic extension of the field $\mathbb{K}$ of characteristic 2 such that all squares of $\mathbb{A}$ are contained in $\mathbb{K}$, or $\mathbb{A}=\mathbb{K}$ of characteristic 2 . Then the action of $\mathrm{PSL}_{3}(\mathbb{A})$ on $\mathbb{P}(V)$ induced by the action 
on $\mathcal{V}_{2}(\mathbb{K}, \mathbb{A})$ has a unique nontrivial invariant subspace $U$, which is the intersection of all tangent hyperplanes. The induced representation in $U$ is the $(\mathbb{K}, \mathbb{A})$ representation of $\mathrm{PSL}_{3}(\mathbb{A})$, whereas the induced representation in $\mathbb{P}(V) / U$ is the $\mathbb{A}^{2}$-representation of $\mathrm{PSL}_{3}(\mathbb{A})$.

Proof. It follows from Theorem 3.1 that the intersection of all tangent hyperplanes is exactly the space $W$ consisting of the points $\mathbb{K}\left(0,0,0 ; y_{0}, y_{1}, y_{2}\right)$ with $y_{0}, y_{1}, y_{2} \in \mathbb{A}$. We now show that $W$ has no proper nontrivial invariant subspace. So let $W^{\prime} \subseteq W$ be an invariant subspace of $\operatorname{PSL}_{3}(\mathbb{A})$, and suppose $\mathbb{K}\left(0,0,0 ; y_{0}, y_{1}, y_{2}\right) \in W^{\prime}$ for some $y_{0}, y_{1}, y_{2} \in \mathbb{A}$. If $y_{0}=y_{1}=0$, then using the map $\delta_{b}$ for $b \in \mathbb{A}^{\times}$and triality, we deduce as in the previous proof that $W^{\prime}=W$. If $y_{0}=0$ and $y_{1} \neq 0$, then using $\delta_{b}$ and an appropriate conjugate under triality, we deduce that $\mathbb{K}\left(0,0,0 ; 0, b^{2} y_{1}, b y_{2}\right)$ and $\mathbb{K}\left(0,0,0 ; 0, b y_{1}, b y_{2}\right)$ belong to $W^{\prime}$ and hence also $\mathbb{K}\left(0,0,0 ; 0,\left(b^{2}+b\right) y_{1}, 0\right)$. Choosing $b \neq 1$, we are reduced to the previous situation. Similarly, we reduce the situation where all $y_{i}, i=0,1,2$, are nonzero to a situation where at least one of them is zero. Hence, $W^{\prime}=W$ in all cases.

Now we show that there are no other invariant subspaces. For $|\mathbb{K}|=2$, this is a direct computation. So suppose $|\mathbb{K}|>2$. Let $U^{\prime}$ be an invariant subspace with $U^{\prime} \neq W$. Then $U^{\prime}$ certainly contains a point $\mathbb{K}\left(x_{0}, x_{1}, x_{2} ; y_{0}, y_{1}, y_{2}\right)$ with $\left(x_{0}, x_{1}, x_{2}\right) \neq(0,0,0)$. It can then be directly verified that, using translations and their conjugates, we can map such a point onto a point $q=\mathbb{K}\left(0,0,1 ; y_{0}^{\prime}, y_{1}^{\prime}, y_{2}^{\prime}\right)$. Note that, if $\left(y_{0}^{\prime}, y_{1}^{\prime}, y_{2}^{\prime}\right)=(0,0,0)$, then $q$ belongs to $\mathcal{V}_{2}(\mathbb{K}, \mathbb{A})$, and hence the result follows. So, without loss, we may assume that $\left(y_{0}^{\prime}, y_{2}^{\prime}\right) \neq(0,0)$. Using $\delta_{b}$ with $b \in \mathbb{K} \backslash\{0,1\}$, we see that also $\mathbb{K}\left(0,0,1 ; y_{0}^{\prime} b^{-1}, y_{1}^{\prime}, y_{2}^{\prime} b^{-1}\right)$ belongs to $U^{\prime}$ and hence also $\mathbb{K}\left(0,0,0 ; y_{0}^{\prime}\left(1+b^{-1}\right), 0, y_{2}^{\prime}\left(1+b^{-1}\right)\right)$, which belongs to $W$.

So we may assume that $W \subseteq U^{\prime}$. But then $\mathbb{K}(0,0,1 ; 0,0,0) \in \mathcal{V}_{2}(\mathbb{K}, \mathbb{A})$ belongs to $U^{\prime}$, and the result again follows. Hence, $U=W$ is the only nontrivial invariant subspace.

The action of $\tau_{a, b}$ on $\mathbb{P}(V) / U$ is given by $\mathbb{K}\left(x_{0}, x_{1}, x_{2} ; *, *, *\right) \mapsto \mathbb{K}\left(x_{0}+\right.$ $\left.a^{2} X_{2}, x_{1}+b^{2} X_{2}, X_{2} ; *, *, *\right)$, and clearly this and its triality conjugates generate the $\mathbb{A}^{2}$-representation of $\mathrm{PSL}_{3}(\mathbb{A})$. Also, the action of $\tau_{a, b}$ on $U=W$ is given by $\mathbb{K}\left(0,0,0 ; y_{0}, y_{1}, y_{2}\right) \mapsto \mathbb{K}\left(0,0,0 ; y_{0}, y_{1}, y_{2}+a y_{0}+b y_{1}\right)$. Also here, it is clear that this and its triality conjugates generate the $(\mathbb{K}, \mathbb{A})$-representation of $\operatorname{PSL}_{3}(\mathbb{A})$.

The proof of the proposition is complete.

The geometric formulation and interpretation of the previous proposition runs as follows. We leave the details of the proof for the reader since it merely concerns some calculations. For a given elliptic space of a Veronesean cap $(X, \Xi)$, we call the nucleus of it the subspace obtained by intersecting all hyperplanes tangent to the corresponding $X$-ovoid.

Proposition 7.3. Let $\mathbb{A}$ either be a purely inseparable quadratic extension of the field $\mathbb{K}$ of characteristic 2 such that all squares of $\mathbb{A}$ are contained in $\mathbb{K}$, or $\mathbb{A}=\mathbb{K}$ of characteristic 2. Let $W$ be the intersection of all tangent hyperplanes. Then the 
projection of $\mathcal{V}_{2}(\mathbb{K}, \mathbb{A})$ from $W$ onto a complementary plane $\pi$ is an isomorphism of $\mathcal{P}\left(\mathcal{V}_{2}(\mathbb{K}, \mathbb{A})\right)$ onto a subplane of $\pi$ over $\mathbb{A}^{2}$. Also, the set of nuclei of all elliptic spaces of $\mathcal{V}_{2}(\mathbb{K}, \mathbb{A})$ defines a partition of $W$, and three elliptic spaces correspond to confluent lines of $\mathcal{P}_{2}(\mathbb{A})$ if and only if the span of the corresponding nuclei of any two of them contains the third if and only if the span of the corresponding nuclei of any two of them intersects the third nontrivially.

AcKnowledgment. The second and third authors would like to thank the Mathematisches Forschungsinstitut Oberwolfach for providing them with the best possible research environment imaginable during a stay as Oberwolfach Leibniz Fellow (JS) and Visiting Senior Researcher (HVM) in June 2013.

\section{References}

[1] J. André, Über nicht-Desarguessche Ebenen mit transitiver Translationsgruppe, Math. Z. 60 (1954), 156-186.

[2] R. H. Bruck and R. C. Bose, The construction of translation planes from projective spaces, J. Algebra 1 (1964), 85-102.

[3] B. Cooperstein, J. A. Thas, and H. Van Maldeghem, Hermitian Veroneseans over finite fields, Forum Math. 16 (2004), 365-381.

[4] B. De Bruyn and H. Van Maldeghem, Universal and homogeneous embeddings of dual polar spaces of rank 3 defined over quadratic alternative division algebras, J. Reine Angew. Math. (to appear), DOI 10.1515/crelle-2013-0126

[5] P. Dembowski, Finite geometries, Ergebnisse der Mathematik und ihrer Grenzgebiete, 44, Springer-Verlag, Berlin-New York, 1968.

[6] J. W. P. Hirschfeld and J. A. Thas, General Galois geometries, Oxford University Press, Oxford, 1991.

[7] D. R. Hughes and F. C. Piper, Projective planes, Grad. Texts in Math., 6, SpringerVerlag, New York-Berlin, 1973.

[8] I. Kaplansky, Infinite-dimensional quadratic forms admitting composition, Proc. Amer. Math. Soc. 4 (1953), 956-960.

[9] O. Krauss, Geometrische Charakterisierung von Veronesemannigfaltigkeiten, Ph.D. thesis, Braunschweig, February 2014.

[10] F. Mazzocca and N. Melone, Caps and Veronese varieties in projective Galois spaces, Discrete Math. 48 (1984), 243-252.

[11] T. A. Springer and F. Veldkamp, On Hjelmslev-Moufang planes, Math. Z. 107 (1968), 249-263.

[12] J. Schillewaert and H. Van Maldeghem, Hermitian Veronesean caps (N. S. N. Sastry ed.), Buildings, finite geometries and groups, Springer Proc. Math., 10, pp. 175-191, Springer, New York, 2012.

[13] , Quadric Veronesean caps, Bull. Belg. Math. Soc. Simon Stevin 20 (2013), 19-25.

[14] , Projective planes over 2-dimensional quadratic algebras, Adv. Math. 262 (2014), 784-822.

[15] _ Severi varieties over arbitrary fields, preprint.

[16] _ Imbrex geometries, J. Combin. Theory Ser. A 127 (2014), 286-302.

[17] H. Salzmann, D. Betten, T. Grundhöfer, H. Hähl, R. Löwen, and M. Stroppel, Compact projective planes 21, de Gruyter, Berlin, 1995. 
[18] F. Severi, Intorno ai punti doppi impropri di una superficie generale dello spazio a quattro dimensioni, e a suoi punti tripli apparenti, Rend. Circ. Mat. Palermo 15 (1901), 33-51.

[19] J. A. Thas and H. Van Maldeghem, Classification of finite Veronesean caps, European J. Combin. 25 (2004), 275-285.

[20] J. Tits, Le plan projectif des octaves et les groupes exceptionnels $E_{6}$ et $E_{7}$, Acad. Roy. Belg. Bull. Cl. Sci. (5) 40 (1954), 29-40.

[21] J. Tits and R. M. Weiss, Moufang polygons, Springer Monogr. Math., SpringerVerlag, Berlin, 2002.

O. Krauss

Department of Mathematics

TU Braunschweig

Institut für Analysis und Algebra

Braunschweig 38106

Germany

o.krauss@tu-braunschweig.de

H. Van Maldeghem

Department of Mathematics

Ghent University

Krijgslaan 281-S22

B-9000 Ghent

Belgium
J. Schillewaert

Department of Mathematics

Imperial College

South Kensington Campus

London SW7-2AZ

UK

jschillewaert@gmail.com

hvm@cage.ugent.be 